\title{
Quantization of dynamical symplectic reduction
}

\author{
Martin Bojowald 情 and Artur Tsobanjan吨 \\ ${ }^{1}$ Institute for Gravitation and the Cosmos, The Pennsylvania State University, \\ 104 Davey Lab, University Park, PA 16802, USA \\ ${ }^{2}$ King's College, 133 North River Street, Wilkes-Barre, PA 18711, USA
}

\begin{abstract}
A long-standing problem in quantum gravity and cosmology is the quantization of systems in which time evolution is generated by a constraint that must vanish on solutions. Here, an algebraic formulation of this problem is presented, together with new structures and results, which show that specific conditions need to be satisfied in order for well-defined evolution to be possible.
\end{abstract}

\section{Introduction}

When time-reparameterization-invariant dynamical systems are cast as Hamiltonian theories on a symplectic manifold one finds that time evolution and time-reparameterization flows are generated by one and the same phase-space function - the Hamiltonian constraint. The straightforward application of the usual methods of symplectic reduction to such dynamically constrained systems has the undesirable side-effect of also removing their dynamics, and needs to be replaced by dynamical syplectic reduction. This paper describes a method of dynamical reduction for the quantized versions of such systems, where noncommutativity leads to a host of additional complications. However, since dynamically constrained systems are rarely studied outside of canonical approaches to quantum gravity we dedicate most of this introductory section to the review of their classical (i.e. nonquantum) treatment. Our main results and the structure of the rest of this manuscript are outlined at the end of the introduction.

Given a symplectic manifold $(M, \Omega)$ and $C \in C^{\infty}(M)$, the symplectic reduction [1] $M / C$ of $M$ by $C$ is the orbit space of $M \supset M_{C}: C=0$ with respect to the gauge flow $F_{C}(\epsilon)=$ $\exp \left(\epsilon X_{C}\right)$ in $M_{C}$ generated by the Hamiltonian vector field $X_{C}$ of $C, \mathrm{~d} C=\Omega\left(X_{C}, \cdot\right)$. Because $\mathcal{L}_{X_{C}} C=\Omega\left(X_{C}, X_{C}\right)=0$, the flow preserves $M_{C}$, and the orbit space inherits a unique symplectic form $\Omega_{M / C}$ from the presymplectic form $i^{*} \Omega$ on $M_{C}$, where $i: M_{C} \rightarrow M$ is the inclusion of $M_{C}$ in $M$. The set of observables of the constrained system, which solve the constraint equation $C=0$ and are invariant under the gauge flow, is given by $C^{\infty}(M / C)$.

In addition to implementing a constraint $C=0$ by symplectic reduction, physical systems usually require the definition of a dynamical flow. The canonical way is to select a

*e-mail address: bojowald@gravity.psu.edu

${ }^{\dagger}$ e-mail address: arturtsobanjan@kings.edu 
Hamiltonian function $H \in C^{\infty}(M)$ which generates the dynamical flow $F_{H}(t)=\exp \left(t X_{H}\right)$ with the Hamiltonian vector field $X_{H}$ of $H$. A dynamical flow in the presence of a constraint $C=0$ is consistent if it preserves the constraint surface, that is, $X_{H} C=\Omega\left(X_{C}, X_{H}\right)=$ $-\{C, H\}=0$ on $M_{C}$ with the Poisson bracket $\{\cdot, \cdot\}$ defined by $\Omega$. The same condition ensures that the dynamical flow is well-defined on the reduced phase space $M / C$ because it is compatible with the gauge flow: By the Jacobi identity of $\{\cdot, \cdot\}$, a gauge transformation (that is, the diffeomorphism induced by a gauge flow) commutes with the dynamical flow up to a gauge transformation. Since $\{C, H\}=0$ on $M_{C}$, there is a $\lambda \in C^{\infty}(M)$ such that $\{C, H\}=\lambda C$ on $M$, and

$$
\left[X_{C}, X_{H}\right]=\{\{\cdot, H\}, C\}-\{\{\cdot, C\}, H\}=-\{\cdot,\{C, H\}\}=-X_{\{C, H\}}=-X_{\lambda C} .
$$

In systems typically encountered in general relativity or its cosmological models, the dynamical flow is simultaneously a gauge flow. A system is time-reparameterization invariant if, given a solution $f(t)$ of its dynamical flow such that $\mathrm{d} f / \mathrm{d} t=\{f, H\}$ for all $t \in \mathbb{R}, f(T(t))$ is also a solution for any monotonic $T \in C^{\infty}(\mathbb{R})$. Any such $f(T(t))$ can be obtained from $f(t)$ by following the flow generated by the Hamiltonian itself together with a suitable non-zero multiplier $N \in C^{\infty}(\mathbb{R})$ via

$$
\lim _{\epsilon \rightarrow 0} \frac{f(t+\epsilon N(t))-f(t)}{\epsilon}=N(t) \frac{\mathrm{d} f}{\mathrm{~d} t}=\{f, N H\}
$$

Therefore the Hamiltonian function is itself the generator of a gauge flow. Observables are functions on the orbit space of the gauge flow. This orbit space inherits a Poisson structure from $M$, with symplectic leaves given by the level surfaces of $H$ [2]. Adding a constant to $H$ does not change the dynamical flow. Therefore, without loss of generality, we can assume the relevant symplectic leaf to be given by $H=0$, such that the dynamical generator $H$ is also a constraint.

The Hamiltonian of a time-reparameterization invariant system is therefore a constraint, called the Hamiltonian constraint. In order to emphasize its nature as a constraint, we will slightly change notation and refer to a Hamiltonian constraint as $C$. We refer to symplectic reduction with a Hamiltonian constraint as dynamical symplectic reduction. Associated with this process is the following long-standing problem [3, 4]: Any observable $O \in C^{\infty}(M / C)$ on the reduced phase space can be pulled back to a function on $M_{C}: C=0$ using the projection $p: M_{C} \rightarrow M / C$ to the orbit space. By definition, $p^{*} O$ is constant on the orbits, or time independent if $C$ is a Hamiltonian constraint. In the reduced phase space, therefore, there is no recognizable time evolution in a time-reparameterization invariant theory.

Classically, the problem of identifying time evolution in a time-reparameterization invariant system is usually solved by fixing the gauge flow generated by a Hamiltonian constraint. This construction to determine observables and their evolution does not use the reduced phase space. Given a symplectic manifold $(M, \Omega)$ and a Hamiltonian constraint $C \in C^{\infty}(M)$, a gauge fixing of the gauge flow is accomplished by a global incisive section. 
Definition $1 A$ section $\left(L, \Omega_{L}, \iota\right)$ of the gauge flow generated by a constraint $C$ on $(M, \Omega)$ is a symplectic manifold $\left(L, \Omega_{L}\right)$ (called the gauge-fixed phase space) together with an embedding $\iota: L \rightarrow M_{C}$ such that $\Omega_{L}=\iota^{*} i^{*} \Omega$.

A section $\left(L, \Omega_{L}, \iota\right)$ of the gauge flow generated by a constraint $C$ on $(M, \Omega)$ is global if for every $y \in M_{C}$ there is an $x \in L$ such that $y=F_{C}(\epsilon) \iota(x)$ for some $\epsilon$.

A section $\left(L, \Omega_{L}, \iota\right)$ of the gauge flow generated by a constraint $C$ on $(M, \Omega)$ is incisive if, for all $x_{1}, x_{2} \in L, \iota\left(x_{1}\right)=F_{C}(\epsilon) \iota\left(x_{2}\right)$ for some $\epsilon$ implies $x_{1}=x_{2}$.

The pull-back $\iota^{*}: C^{\infty}\left(M_{C}\right) \rightarrow C^{\infty}(L)$ maps functions on the constraint surface $M_{C}$ to gauge-fixed observables on $L$.

Proposition 1 If $\left(L, \Omega_{L}, \iota\right)$ is a global incisive section of the gauge flow of $C$ on $(M, \Omega)$, the gauge-fixed phase space $\left(L, \Omega_{L}\right)$ is symplectomorphic to the reduced phase space $\left(M / C, \Omega_{M / C}\right)$.

Proof: Since a global incisive section intersects each gauge orbit exactly once, there is a bijection between $L$ and the reduced phase space. The symplectomorphism property can then be shown in local coordinates: Locally, $C$ can be used as a coordinate in a neighborhood around a given point $x \in M_{C} \subset M$. We use the gauge flow $F_{C}(\epsilon): x \mapsto$ $x_{\epsilon} \in M_{C}$ to introduce a second coordinate $z$ such that $z(x)=0$ and $z\left(x_{\epsilon}\right)=\epsilon$. The two functions $C$ and $z$ are canonically conjugate: $\{z, C\}=X_{C} z=\mathrm{d} z / \mathrm{d} \epsilon=1$. By Darboux' theorem, there are $\operatorname{dim} M-2$ additional local coordinates $q_{j}$ and $p_{k}$, such that

$$
\Omega_{M}=\mathrm{d} z \wedge \mathrm{d} C+\sum_{j=1}^{\frac{1}{2} \operatorname{dim} M-1} \mathrm{~d} q_{j} \wedge \mathrm{d} p_{j} .
$$

Since $0=\left\{q_{j}, z\right\}=\partial q_{j} / \partial C$ and $0=\left\{p_{j}, z\right\}=\partial p_{j} / \partial C, q_{j}$ and $p_{k}$ together with $z$ define a local coordinate system on $M_{C}$.

On $M_{C}, i^{*} \Omega=\sum_{j=1}^{\frac{1}{2} \operatorname{dim} M-1} \mathrm{~d} q_{j} \wedge \mathrm{d} p_{j}$ is a presymplectic form. Local intervals of gauge orbits of $C$ are the coordinate lines of $z$. Therefore, $q_{j}$ and $p_{k}$ are local coordinates on the reduced phase space, with symplectic form $\Omega_{M / C}=\sum_{j=1}^{\frac{1}{2} \operatorname{dim} M-1} \mathrm{~d} q_{j} \wedge \mathrm{d} p_{j}$. In order for $\iota^{*} i^{*} \Omega$ to be symplectic, any section of the gauge flow must locally be of the form $\iota: y \mapsto(s(y), z(s(y)))$ with a canonical transformation $s: y \mapsto\left(q_{J}, p_{k}\right)$ and a smooth function $z\left(q_{j}, p_{k}\right)$. Therefore, $\Omega_{L}=\iota^{*} i^{*} \Omega=s^{*} \sum_{j=1}^{\frac{1}{2} \operatorname{dim} M-1} \mathrm{~d} q_{j} \wedge \mathrm{d} p_{j}=s^{*} \Omega_{M / C}$.

An incisive section $\left(L, \Omega_{L}, \iota\right)$ evolves in $M$ if there is a 1-parameter family of incisive sections $\left(L, \Omega_{L}, \iota_{t}\right), t \in\left(t_{1}, t_{2}\right) \subset \mathbb{R}$, such that $\iota=\iota_{t_{0}}$ for some $t_{0} \in\left(t_{1}, t_{2}\right)$, and $L \times\left(t_{1}, t_{2}\right) \rightarrow$ $\mathcal{U},(y, t) \mapsto \iota_{t}(y)$ is a diffeomorphism to an open submanifold $\mathcal{U} \subset M_{C}$. For each value of $t \in\left(t_{1}, t_{2}\right)$ the hypersurface $\iota_{t}(L) \subset M_{C}$ plays the role of a surface of a fixed value of time. With this structure in place, any function $f \in C^{\infty}\left(M_{C}\right)$ can be viewed as evolving in time along any given gauge orbit by tracing it along the intersection between the orbit and the constant-time surfaces.

$$
f_{[x]}(t)=f\left([x] \cap \iota_{t}(L)\right),
$$


where $[x]$ is the gauge orbit passing through some $x \in M_{C}$.

This time evolution takes place on the pre-symplectic manifold $M_{C}$ which is not the usual setting for describing a dynamical physical system. Moreover, it is not a good starting point for standard quantization as functions on $M_{C}$ do not have a well-defined Poisson bracket due to the degeneracy of $i^{*} \Omega$. However, an evolving incisive section defines a family of functions in $C^{\infty}(\mathcal{U})$, namely those that are constant along the curves traced by points in $L$ from one section to the next $x(t)=\iota_{t}(y)$ for a fixed $y \in L$. This family of functions can be arbitrarily extended to the entirety of $M_{C}$ :

Definition $2 A$ subset $\mathcal{F} \subset C^{\infty}\left(M_{C}\right)$ is fashionable with respect to an evolving incisive section $\left(L, \Omega_{L}, \iota_{t}\right)$ if for all $t, t^{\prime} \in\left(t_{1}, t_{2}\right)$ the map $\iota_{t}^{*}: \mathcal{F} \rightarrow C^{\infty}(L)$ is a bijection and $\iota_{t}^{*} f=\iota_{t^{\prime}}^{*} f$ for all $f \in \mathcal{F}$.

Given a choice of fashionables, each function on the symplectic manifold $L$ corresponds to an evolving observable on $M_{C}$ and conversely, the set of evolving observables $\mathcal{F}$ inherits a Poisson bracket from the symplectic structure on $L$.

This notion of evolution has no known analog in the reduced phase space. In practice it is usually implemented through deparameterization [5, 6, 7, 8, 9, provided the constraint surface admits a factorization of the form $M_{C} \cong \iota(L) \times \mathbb{R} \ni(\iota(x), Z)$ with a global coordinate $Z \in \mathbb{R}$ such that $\{Z, C\} \neq 0$. Then the map $\iota_{t}: L \rightarrow M_{C}, x \mapsto(x, t)$ defines a family of global incisive sections. Evolution defined by this family of sections and $\mathcal{F}$, the $Z$-independent functions on $M_{C}$, is called global relational evolution with respect to $Z$.

Example: Let $M=\mathbb{R}^{2(n+1)} \ni\left(Z, E, q_{1}, p_{1}, \ldots q_{n}, p_{n}\right)$ with

$$
\Omega=\mathrm{d} Z \wedge \mathrm{d} E+\sum_{i=1}^{n} \mathrm{~d} q_{i} \wedge \mathrm{d} p_{i}
$$

and a constraint $C=E+h\left(Z, q_{i}, p_{i}\right)$ linear in $E$. The constraint surface here consists of points with coordinates $\left(Z,-h\left(q_{i}, p_{i}\right), q_{i}, p_{i}\right)$, so that $\left(Z, q_{i}, p_{i}\right)$ serve as coordinates on $M_{C}$. The choice $L=\mathbb{R}^{2 n} \ni\left(Q_{1}, P_{1}, \ldots Q_{n}, P_{n}\right)$ then leads to global incisive deparameterized sections via $\iota_{t}:\left(Q_{i}, P_{i}\right) \mapsto\left(t, Q_{i}, P_{i}\right) \in M_{C}$. Since for $C^{\infty}\left(M_{C}\right) \ni f=f\left(Z, q_{i}, p_{i}\right)$ under this family of embeddings $\left(\iota_{t}^{*} f\right)\left(Q_{i}, P_{i}\right)=f\left(t, Q_{i}, P_{i}\right)$, the corresponding fashionables consist precisely of the functions that do not depend on $Z$. Since the Hamiltonian vector field of $E$ generates translations in $Z$ and hence shifts from $\iota_{t}(L)$ to $\iota_{t^{\prime}}(L)$, the set of fashionables correspond to the Poisson commutant $E^{\prime}=\left\{f \in C^{\infty}(M):\{f, E\}=0\right\}$ of $E$ pulled back to $M_{C}$. Relational evolution with respect to $Z$ is identical with Hamiltonian evolution in $L$ generated by $H_{t}\left(Q_{i}, P_{i}\right)=h\left(t, Q_{i}, P_{i}\right)$ : The gauge flow $F_{C}(\epsilon)$ on $M$ maps a function $g \in C^{\infty}(M)$ to $g_{\epsilon}=F_{C}(\epsilon)^{*} g$. In an infinitesimal version, $\delta g / \delta \epsilon:=\lim _{\epsilon \rightarrow 0}\left(g_{\epsilon}-g\right) / \epsilon$ is given by

$$
\frac{\delta g}{\delta \epsilon}=\{g, C\}=\frac{\partial g}{\partial Z}+\{g, h\}
$$

Specifically,

$$
\frac{\delta q_{i}}{\delta \epsilon}=\frac{\partial h}{\partial p_{i}} \quad, \quad \frac{\delta p_{i}}{\delta \epsilon}=-\frac{\partial h}{\partial q_{i}} \quad, \quad \frac{\delta Z}{\delta \epsilon}=1 .
$$


This pulls back to $L$ as

$$
\frac{\delta Q_{i}}{\delta \epsilon}=\frac{\partial H_{t}}{\partial P_{i}} \quad, \quad \frac{\delta P_{i}}{\delta \epsilon}=-\frac{\partial H_{t}}{\partial Q_{i}} .
$$

For a function on $L$, we have

$$
\begin{aligned}
\frac{\delta f}{\delta \epsilon} & =\lim _{\epsilon \rightarrow 0} \frac{f\left(Q_{i}+\epsilon \delta Q_{i} / \delta \epsilon, P_{i}+\epsilon \delta P_{i} / \delta \epsilon\right)-f\left(Q_{i}, P_{i}\right)}{\epsilon} \\
& =\sum_{i=1}^{n}\left(\frac{\partial f}{\partial Q_{i}} \frac{\delta Q_{i}}{\delta \epsilon}+\frac{\partial f}{\partial P_{i}} \frac{\delta P_{i}}{\delta \epsilon}\right)=\{f, H\}_{L}
\end{aligned}
$$

computed precisely according to Hamilton's equations on $L$.

The quantization of a reduced phase space exists in the sense of deformation quantization [10, 11] à la Fedosov or Kontsevich [12. On the other hand, dynamical symplectic reduction is usually quantized only for deparameterized systems as in the immediately preceding example, using a standard Hilbert-space quantization of $L$ on which the reduced Hamiltonian $H_{t}\left(Q_{i}, P_{i}\right)$ is represented as an operator. In such examples, quantum evolution exists and is unitary, but there are long-standing problems when one tries to extend this notion to more complicated constrained systems in which no global analog of $Z$ exists [3, 4. For instance, given a constraint quadratic in $E$ on the same phase space as in the example, $\{Z, C\} \propto E$ may become zero along a gauge orbit such that $Z=$ const no longer defines a gauge section.

Heuristically, if there is no global analog of $Z$, evolution cannot be represented by a family of unitary operators on a Hilbert space. Based on this observation, we diagnose the main problem of standard approaches of deparameterization as an over-reliance on Hilbertspace representations. In order to solve this problem, we initiate a theory of algebraic sections as an algebraic quantization of classical gauge sections in systems with a single constraint. By generalizing crucial steps of deparameterization and keeping them strictly at the level of algebras of observables, utilizing factor spaces of algebras instead of kernels of constraint operators acting on a Hilbert space, we define a quantum version of Proposition 1 and derive several necessary conditions that must be met by the constrained system in order for it to be dynamically reducible. While it remains difficult to find sufficient conditions for such a result, the usefulness of our necessary conditions is demonstrated by their restrictive nature in a specific example relevant to cosmology provided at the end of this paper.

After setting the stage in Section 2, in Section 3] we define algebraic qantization of dynamical symplectic reduction and prove several properties of the resulting quantum evolution on an algebra of observables. For deparameterizable systems, which can be quantized by well-established means as representations on a fixed Hilbert space, our algebraic results provide a more general treatment because they apply to all possible choices of the Hilbert space. Moreover, our construction applies to non-deparameterizable systems, even though the results in that case are less specific than for deparameterizable systems. Several results and examples in Section 4 will demonstrate the non-trivial nature of our constructions. 


\section{Preliminary constructions}

To set the stage, we introduce in this section more details of the systems of interest, standard procedures to quantize them as well as their limitations, and basic algebraic ingredients to be used in our main results.

\subsection{Systems of interest}

Quantum cosmology presents several versions of singly constrained systems, resulting from the generally covariant theory of general relativity from which their classical analogs are derived. Since space appears to be homogeneous and isotropic over long distances, one can approximately describe the expansion of the universe by a single time-dependent function, the scale factor $a(t)$, subject to a single constraint. The latter is derived from the Friedmann equation

$$
\frac{1}{a^{2}}\left(\frac{\mathrm{d} a}{\mathrm{~d} t}\right)^{2}=\frac{8 \pi}{3} \rho(a)
$$

with the matter energy density $\rho(a)$ (written in units such that Newton's constant and the speed of light equal one). In canonical form, a multiple of the expansion rate $a^{-1} \mathrm{~d} a / \mathrm{d} t$ is canonically conjugate to the volume $V=a^{3}$,

$$
p_{V}=-\frac{1}{4 \pi a} \frac{\mathrm{d} a}{\mathrm{~d} t}
$$

such that $\left\{V, p_{V}\right\}=1$. The canonical Friedmann equation,

$$
-6 \pi V p_{V}^{2}+E(V)=0
$$

can be written as a constraint equation which equates the matter energy $E(V)=V \rho\left(V^{1 / 3}\right)$ with a polynomial function of the volume and the expansion rate. For non-relativistic matter (dominant at late times in the universe), the energy density changes only by dilution in the expanding universe, such that $\rho \propto 1 / V$, or $E(V)=E$ constant. The resulting constraint

$$
C=-6 \pi V p_{V}^{2}+E,
$$

polynomial in $V$ and $p_{V}$ and linear in $E$, is a prototype of a large set of models that have been studied to understand quantum evolution in covariant systems.

The dependence of $C$ on $V$ and $p_{V}$ varies according to the cosmological model of interest. Moreover, there are additional anisotropy degrees of freedom if one drops the assumption of spatial isotropy. Many models of this type are known to have chaotic dynamics [13], such that there is usually no practical access to the reduced phase space $M / C$. Another

set of models is motivated by modified gravity, for instance the application of a variety of quantization procedures, which may replace the Heisenberg algebra generated by $V$ and $p_{V}$ by a different Lie algebra. Examples of this type have been produced by models of loop quantum cosmology [14, 15], many of which can be formulated based on the Lie algebra $\operatorname{sl}(2, \mathbb{R})$ instead of the Heisenberg algebra generated by $V$ and $p_{V}[16,17,18,19,20$. 
(If there are constraints in addition to $C$, which however do not contribute to the dynamical flow, one can combine standard symplectic reduction with dynamical symplectic reduction. For instance, in some cosmological models, the single variable $V$ could be replaced by a pair $\left(V_{1}, V_{2}\right)$ with momenta $\left(p_{V_{1}}, p_{V_{2}}\right)$, subject to rotational symmetry in the plane. A phase-space formulation would then introduce a non-dynamical constraint $J=V_{1} p_{V_{1}}-V_{2} p_{V_{2}}=0$ given by angular momentum in the plane. If the dynamical constraint $C$ extended to the pair $\left(V_{1}, V_{2}\right)$ is rotationally symmetric, it has vanishing Poisson bracket with the non-dynamical constraint, $\{C, J\}=0$, at least on the constraint surface $J=0$ of $J$. In this example, and in many others of interest to gravity and cosmology, the Hamiltonian constraint is accompanied by finitely many additional constraints. In such cases standard symplectic reduction of $J$ can easily be combined with dynamical symplectic reduction of $C$, also at the quantum level. A further generalization to field theories would be required if one were to include perturbations around isotropic cosmological models in order to describe inhomogeneous matter fields on an expanding space-time background. Because time coordinates can be changed locally in general relativity, there would then be not only an infinite number of degrees of freedom, but also an infinite number of dynamical constraints, one per point in space. Although such systems are certainly important for modern cosmology, investigations of deparameterization in this context have remained in their infancy. In particular, there seems to be no consensus so far on the physical properties that should be required of deparameterization in the presence of multiple dynamical constraints. In what follows, we will therefore consider only the case of a single dynamical constraint.)

In order to introduce evolution in the constrained picture, it is common to consider the constant $E$ as the momentum of a canonical variable $Z$ on which the constraint does not depend. The constraint can then be quantized on the kinematical Hilbert space $\mathcal{H}_{\text {kin }}=$ $L^{2}(\mathbb{R}, \mathrm{d} \lambda) \otimes \mathcal{H}_{\mathcal{B}}$ whose first factor is the Schrödinger representation of the Heisenberg algebra generated by $E$ and $Z$, such that $E=i \hbar \partial / \partial \lambda$, while $\mathcal{H}_{\mathcal{B}}$ is a unitary representation of the algebra $\mathcal{B}$ generated by the original canonical variables, such as the Heisenberg algebra generated by $V$ and $p_{V}$.

If $C$ does not depend on $Z$, as in (11), the standard procedure of deparameterization, first suggested by Dirac [5] and applied to quantum cosmology starting with [21], can be used to quantize the dynamically constrained system: The constraint $C$ is represented as an operator on $\mathcal{H}_{\text {kin }}$ such that

$$
C \psi=i \hbar \frac{\partial \psi(\lambda)}{\partial \lambda}-H \psi(\lambda)
$$

where $H$ is a self-adjoint representation of the $\left(V, p_{V}\right)$-dependent contribution to $C$ on $\mathcal{H}_{\mathcal{B}}$. Zero eigenvectors of $C$ are therefore given by

$$
\psi(\lambda)=\exp (-i \lambda H / \hbar) \psi_{0}
$$

with arbitrary $\psi_{0} \in \mathcal{H}_{\mathcal{B}}$ as an "initial state" with respect to evolution in $\lambda$. Because $U(\lambda)=\exp (-i \lambda H / \hbar)$ is unitary, $\psi(\lambda)$ is not normalizable in $\mathcal{H}_{\text {kin }}$, such that zero is in the continuous part of the spectrum of $C$. 
In order to introduce a Hilbert-space structure on the solutions $\psi(\lambda)$, we again make use of the unitarity of $U(\lambda)$ and define a new inner product on the solution space by recycling the inner product $(\cdot, \cdot)_{\mathcal{B}}$ on $\mathcal{H}_{\mathcal{B}}$ : The physical inner product

$$
(\psi(\lambda), \phi(\lambda))_{\mathrm{phys}}=\left(\psi_{0}, \phi_{0}\right)_{\mathcal{B}}
$$

turns the solution space into the physical Hilbert space $\mathcal{H}_{\text {phys }}$ (which is not a subspace of $\left.\mathcal{H}_{\text {kin }}\right)$. Unitarity of $U(\lambda)$ implies that the inner product does not depend on the choice of an initial $\lambda$-time:

$$
\left(\psi\left(\lambda-\lambda_{0}\right), \phi\left(\lambda-\lambda_{0}\right)\right)_{\mathrm{phys}}=\left(\psi_{0}, U\left(-\lambda_{0}\right)^{\dagger} U\left(-\lambda_{0}\right) \phi_{0}\right)_{\mathcal{B}}=\left(\psi_{0}, \phi_{0}\right)_{\mathcal{B}} .
$$

Moreover, any operator that commutes with $U(\lambda)$, called a Dirac observable, has a unique representation on $\mathcal{H}_{\text {phys }}$ However, it is usually hard to compute Dirac observables or to show the existence of a large-enough set, and they do not evolve because, by definition, they commute with $U(\lambda)$.

In order to introduce an evolution picture on $\mathcal{H}_{\text {phys }}$, one often represents any operator $B \in \mathcal{B}$ by fixing an initial time, such that the action of $B$ on $\psi_{0} \in \mathcal{H}_{\mathcal{B}}$ can be used. However, this representation is not natural because choosing a different initial time $\lambda_{0}$, such that $\psi_{0}$ is replaced by $U\left(-\lambda_{0}\right) \psi_{0}$, leads to a different (but unitarily equivalent) representation unless $B$ commutes with $U\left(-\lambda_{0}\right)$. With this construction, evolution is realized by the $\lambda$-dependent expectation values

$$
(\psi(\lambda), B \psi(\lambda))_{\mathrm{phys}}=\left(\psi_{0}, B \psi_{0}\right)_{\mathcal{B}} .
$$

The compution of Dirac observables can therefore be avoided if one accepts the dependence of representations on the choice of an initial time.

However, the applicability of these constructions is limited because they rely on timeindependent constraints which do not depend on the variable $Z$ canonically conjugate to $E$. Generic matter models in quantum cosmology and other fields require such a dependence. For instance, in order to determine evolution with respect to different choices of time coordinates, one would have to interpret the gauge flows generated by $N C$ with some $N \in \mathcal{A}$, rather than $C$ itself, and $N$ may well depend on $Z$ in cases of interest. Moreover, relativistic matter systems imply an energy density quadratic in $E$ rather than linear, a prominent example given by a homogeneous scalar field $Z$ (such as the inflaton often assumed in early-universe cosmology) with energy density

$$
\rho_{\text {scalar }}=\frac{1}{2} \frac{E^{2}}{V^{2}}+W(Z)
$$

where the function $W(Z)$ is the scalar potential. The resulting constraint,

$$
-12 \pi V^{2} p_{V}^{2}+E^{2}+2 V^{2} W(Z)=0,
$$

is still polynomial in $V$ and $p_{V}$ but quadratic in $E$. If $W(Z)=W$ is constant, one can often "take a square root" and replace the constraint for a scalar energy density with a 
constraint linear in $E$ by factorization,

$$
\left(E-\sqrt{2} V \sqrt{6 \pi p_{V}^{2}-W}\right)\left(E+\sqrt{2} V \sqrt{6 \pi p_{V}^{2}-W}\right)=0,
$$

followed by selecting one of the two parentheses as a "linearized" constraint. To the new, linear constraint one can then apply deparameterization as sketched above [6]. However, constant $W(Z)=W$ is not generic within the set of physically motivated models, and for non-constant $W(Z)$ any factorization is non-trivial because $[W(Z), E] \neq 0$.

Here, we propose an alternative way of reducing a quantum system with a Hamiltonian constraint to a dynamical system, that solves most of these problems and also reveals the non-trivial nature of introducing a well-defined evolution picture. We completely avoid the construction of a physical Hilbert space $\mathcal{H}_{\text {phys }}$ distinct from the original, kinematical Hilbert space $\mathcal{H}_{\text {kin }}$. (Nevertheless, we will show that, if desired, a physical Hilbert space can be derived from a subset of our ingredients using the Gelfand-Naimark-Segal construction.) This feature brings our constructions closer to a relativistic setting which seems violated in the construction described above in which time is an operator represented only on $\mathcal{H}_{\text {kin }}$, while all other observables are represented on $\mathcal{H}_{\text {phys }}$. Our approach is based on an algebraic notion of quantum states.

\subsection{Algebraic states}

The set of observables of a quantum system is given by the $*$-invariant elements of a complex, unital $*$-algebra $\mathcal{A}$. In this paper, we assume that $\mathcal{A}$ is associative. (This assumption rules out some physical systems, such as magnetic monopole densities [22, 23], which however are usually considered exotic.) Our main examples will be enveloping algebras of Lie algebras, which we assume to be represented on a kinematical Hilbert space as unbounded operators. These algebras carry a useful topology, introduced as the $\rho$-topology in [24].

Physical states of the quantum system defined by $\mathcal{A}$ are normalized positive linear functionals $\omega: \mathcal{A} \rightarrow \mathbb{C}$, such that $\omega(\mathbf{1})=1$ and

$$
\omega\left(A A^{*}\right) \geq 0 \text { for all } A \in \mathcal{A} .
$$

According to Theorem 1 in [24], such functionals are continuous in the $\rho$-topology of $\mathcal{A}$. The condition that $\omega\left(A A^{*}\right)$ is real for all $A \in \mathcal{A}$ implies that a physical state is real $\omega(A)=\overline{\omega\left(A^{*}\right)}$. In addition, the stronger inequality condition leads to the Cauchy-Schwarz inequality

$$
\left|\omega\left(A B^{*}\right)\right|^{2} \leq\left|\omega\left(A A^{*}\right)\right|\left|\omega\left(B B^{*}\right)\right| \quad \text { for all } A, B \in \mathcal{A} ;
$$

see for instance [25].

As we will see, intermediate stages of quantum symplectic reduction require a weaker notion of states which are not completely positive. We begin with

Definition 3 The set of kinematical states $\Gamma$ on a unital $*$-algebra $\mathcal{A}$ is the set of continuous normalized linear functionals $\omega: \mathcal{A} \rightarrow \mathbb{C}$, such that $\omega(\mathbf{1})=1$. 
Given the normalization condition, $\Gamma$ is not a vector space, but it is closed with respect to normalized sums: for any integer $N \geq 1$, states $\omega_{1}, \ldots, \omega_{N} \in \Gamma$ and complex numbers $a_{1}, \ldots, a_{N}, \sum_{j=1}^{N} a_{j} \omega_{j} \in \Gamma$ if $\sum_{j=1}^{N} a_{j}=\overline{1}$.

Definition $4 A$ dynamical flow on $\mathcal{A}$ is a one-parameter family of derivations $\vec{D}_{t}:(a, b) \times$ $\mathcal{A} \rightarrow \mathcal{A}$, where $(a, b) \subset \mathbb{R}$, which is compatible with the *-structure on $\mathcal{A}-\left(\vec{D}_{t} A\right)^{*}=\vec{D}_{t} A^{*}$ for all $A \in \mathcal{A}$ - and such that $\omega\left(\vec{D}_{t} A\right)$ is continuously differentiable with respect to $t$ for all $\omega \in \Gamma$.

Given a dynamical flow $\vec{D}_{t}$ on $\mathcal{A}$, the time evolution of a kinematical state $\omega \in \Gamma$ is a $\operatorname{map}(a, b) \times \mathcal{A} \rightarrow \mathbb{C},(t, A) \mapsto \omega_{t}(A)$ such that $\omega_{t}$ is a kinematical state and

$$
\frac{\mathrm{d}}{\mathrm{d} t} \omega_{t}(A)=\omega_{t}\left(\vec{D}_{t} A\right)
$$

for all $t \in(a, b)$, with initial conditions $\omega_{t_{0}}=\omega$ for some $t_{0} \in(a, b)$.

In order to make sure that a state has a unique time evolution (or a unique gauge flow in what follows), we will assume that, for all algebras we consider, a differential equation of the form (3) has a unique solution with the specified initial condition. Standard results do not necessarily apply because our differential equations, though linear, are, in general, formulated on an infinite-dimensional space and may have time-dependent coefficients. (Although we will not pursue a formal proof of existence and uniqueness of solutions, we note that time evolution in systems of interest in physics is usually obtained as a unique Dyson series on a Hilbert space; see for instance [26].)

Lemma 1 If $\omega \in \Gamma$ is a kinematical state, its time evolution with respect to $\vec{D}_{t}, t \in(a, b)$, returns a kinematical state for any $t \in(a, b)$.

Proof: By definition, a derivation satisfies

$$
\vec{D}_{t}(A B)=\vec{D}_{t}(A) B+A \vec{D}_{t}(B)
$$

for all $A, B \in \mathcal{A}$. Choosing $B=\mathbf{1}$, we have $\vec{D}_{t}(A)=\vec{D}_{t}(A)+A \vec{D}_{t}(\mathbf{1})$ for all $A \in \mathcal{A}$. It follows that $\vec{D}_{t}(\mathbf{1})=0$, whence $\mathrm{d} \omega_{t}(\mathbf{1}) / \mathrm{d} t=0$ for all $t$. Therefore, $\omega_{t}(\mathbf{1})=1$ for all $t$.

Lemma 2 If $\omega \in \Gamma$ is positive, its time evolution is positive.

Proof: To prove that $\omega_{t}\left(A A^{*}\right) \geq 0$ continues to hold along the flow, it is sufficient to show that (i) $\omega_{t}\left(A A^{*}\right)$ is real for all $t$ and (ii) $\mathrm{d} \omega_{t}\left(A A^{*}\right) / \mathrm{d} t$ is non-negative whenever $\omega_{t}\left(A A^{*}\right)=0$.

To prove (i), for each $A \in \mathcal{A}$ define a function of $t$ via $f_{A}(t)=\omega_{t}\left(A A^{*}\right)-\overline{\omega_{t}\left(A A^{*}\right)}$ on $\Gamma$, so that $\omega_{t}\left(A A^{*}\right)$ is real iff $f_{A}(t)=0$. Suppose all of the functions $f_{A}\left(t^{\prime}\right)=0$ for some 
$t^{\prime} \in(a, b)$, then $\omega_{t^{\prime}}\left(A A^{*}\right)$ is real for all $A \in \mathcal{A}$, which implies $\omega_{t^{\prime}}(A)=\overline{\omega_{t^{\prime}}\left(A^{*}\right)}$, and we get

$$
\begin{aligned}
& \left.\frac{\mathrm{d}}{\mathrm{d} t} \omega_{t}\left(A A^{*}\right)\right|_{t=t^{\prime}}=\left.\omega_{t}\left(\vec{D}_{t}\left(A A^{*}\right)\right)\right|_{t=t^{\prime}}=\omega_{t^{\prime}}\left(\left(\vec{D}_{t^{\prime}} A\right) A^{*}\right)+\omega_{t^{\prime}}\left(A\left(\vec{D}_{t^{\prime}} A^{*}\right)\right) \\
& =\omega_{t^{\prime}}\left(\left(\vec{D}_{t^{\prime}} A\right) A^{*}\right)+\omega_{t^{\prime}}\left(\left[\left(\vec{D}_{t^{\prime}} A\right) A^{*}\right]^{*}\right) \\
& =\omega_{t^{\prime}}\left(\left(\vec{D}_{t^{\prime}} A\right) A^{*}\right)+\overline{\omega_{t^{\prime}}\left(\left(\vec{D}_{t^{\prime}} A\right) A^{*}\right)} \\
& =2 \operatorname{Re}\left[\omega_{t^{\prime}}\left(\left(\vec{D}_{t^{\prime}} A\right) A^{*}\right)\right]=2 \operatorname{Re}\left[\overline{\omega_{t^{\prime}}\left(\left(\vec{D}_{t^{\prime}} A\right) A^{*}\right)}\right]=\left.\frac{\mathrm{d}}{\mathrm{d} t} \overline{\omega_{t}\left(A A^{*}\right)}\right|_{t=t^{\prime}},
\end{aligned}
$$

which means that $\mathrm{d} f_{A}(t) / \mathrm{d} t=0$ at $t=t^{\prime}$. Since $\omega_{t_{0}}=\omega$ is positive, we have the initial conditions $f_{A}\left(t_{0}\right)=0$ for all $A \in \mathcal{A}$. We see that $\left\{f_{A}(t)=0, \forall t \in(a, b)\right\}_{A \in \mathcal{A}}$ satisfies the first-order ordinary differential equation system induced by the dynamical flow and matches the given set of initial conditions. As previously discussed, here we assume such solutions to the dynamical flow to be unique. Therefore $\omega_{t}\left(A A^{*}\right)$ is real for all $t \in(a, b)$.

To prove (ii) we use the above result and assume that the inequality holds at $t=t^{\prime}$.

$$
\begin{aligned}
\left.\left|\frac{\mathrm{d}}{\mathrm{d} t} \omega_{t}\left(A A^{*}\right)\right|_{t=t^{\prime}}\right|^{2} & =4\left|\operatorname{Re}\left[\omega_{t^{\prime}}\left(\left(\vec{D}_{t^{\prime}} A\right) A^{*}\right)\right]\right|^{2} \\
& \leq 4\left|\omega_{t^{\prime}}\left(\left(\vec{D}_{t^{\prime}} A\right) A^{*}\right)\right|^{2} \\
& \leq 4 \omega_{t^{\prime}}\left(A^{*} A\right) \omega_{t^{\prime}}\left(\left(\vec{D}_{t^{\prime}} A\right)\left(\vec{D}_{t^{\prime}} A\right)^{*}\right) .
\end{aligned}
$$

Since $\omega_{t^{\prime}}\left(A^{*} A\right) \in \mathbb{R}, \omega_{t^{\prime}}\left(A^{*} A\right)=\omega_{t^{\prime}}\left(A A^{*}\right)$, and the expression on the right is zero if $\omega_{t^{\prime}}\left(A A^{*}\right)=0$.

\subsection{Constrained quantization}

Our results in Sections 3 and 4 apply to a specific type of constrained systems relevant for quantizations of dynamical symplectic reduction.

Definition 5 A singly constrained quantum system is a complex, unital $*$-algebra $\mathcal{A}$ together with a constraint $C \in \mathcal{A}$ such that

1. $C^{*}=C$,

2. $C$ does not have a left-inverse in $\mathcal{A}$, and

3. $C$ is not a divisor of zero.

Remark: If $C$ is a right divisor of zero, then by property 1 it is also a left divisor of zero and vice versa, in which case there is an $X \in \mathcal{A}$ such that $C X=0$. Using a representation of 
$\mathcal{A}$ on the kinematical Hilbert space, any vector in the image of $X$ is then a zero eigenvector of $C$ in the discrete spectrum, and one can simply solve the constraint $C=0$ by restriction to the zero eigenspace. Here we are primarily concerned with the more complicated case of zero in the continuous part of the spectrum of $C$, which is also most relevant for dynamical symplectic reduction of examples discussed in Section 2.1.

Definition 6 The algebra of Dirac observables of a singly constrained quantum system $(\mathcal{A}, C)$ is the commutant of $C$ in $\mathcal{A}$ :

$$
\mathcal{A}_{\text {obs }}=C^{\prime}=\{A \in \mathcal{A}:[A, C]=0\} .
$$

The space of physical states of a singly constrained quantum system is given by the space $\Gamma\left(\mathcal{A}_{\mathrm{obs}}\right)$ of normalizaed positive linear functionals on $\mathcal{A}_{\mathrm{obs}}$.

Lemma $3 \mathcal{A}_{\text {obs }}$ is a unital *-subalgebra of $\mathcal{A}$.

Proof: $\quad$ Defined as the commutant of $C, \mathcal{A}_{\mathrm{obs}}$ is a subalgebra. Since $[\mathbf{1}, C]=0$ and $\left[A^{*}, C\right]=-\left[A, C^{*}\right]^{*}=-[A, C]^{*}=0$ if $A \in \mathcal{A}_{\mathrm{obs}}$, using $C^{*}=C$, it is a unital $*$-subalgebra.

Definition 7 A kinematical state $\omega \in \Gamma$ is a solution of the constraint $C$ if $\omega(A C)=0$ for all $A \in \mathcal{A}$. The constraint surface $\Gamma_{C} \subset \Gamma$ is the subset of all solutions of $C$, closed with respect to normalized sums.

Remark: Since we have assumed that $C$ is without left-inverse in $\mathcal{A}, \mathcal{A} C \subset \mathcal{A}$ is a strict subalgebra without unit. The condition $\omega(A C)=0$ is therefore consistent with normalization of kinematical states. If zero is in the continuous spectrum, no normalized positive states exist in $\Gamma_{C}$. In such a case it is common to drop the normalization condition and work with distributions instead of state vectors in a Hilbert space. Here, we instead retain the normalizability condition and relax positivity. Our results (such as Corollary2 in Section 3.3) imply the existence of kinematical states in $\Gamma_{C}$ for singly constrained systems.

The constraint $C$ in a singly constrained quantum system induces a gauge flow:

Definition 8 Two kinematical states $\psi, \omega \in \Gamma$ are $C$-equivalent, $\omega \sim_{C} \psi$, if there exist a positive integer $M$ together with $A_{1}, A_{2}, \ldots, A_{M} \in \mathcal{A}$ and $\lambda_{1}, \lambda_{2}, \ldots \lambda_{M} \in \mathbb{R}$, such that

$$
\psi=S_{A_{1} C}\left(\lambda_{1}\right) S_{A_{2} C}\left(\lambda_{2}\right) \ldots S_{A_{M} C}\left(\lambda_{M}\right) \omega
$$

where for $A \in \mathcal{A}$ and $\lambda \in \mathbb{R}$, the flow $S_{A}(\lambda): \Gamma \rightarrow \Gamma$ is defined by $S_{A}(0)=\mathrm{id}$ and

$$
i \hbar \frac{\mathrm{d}}{\mathrm{d} \lambda}\left(S_{A}(\lambda) \omega(B)\right)=S_{A}(\lambda) \omega([B, A]) .
$$


Since $B \mapsto[B, A]$ is a derivation, $S_{A}(\lambda)$ is well-defined by Lemma 1, By analogy with classical reduction, we refer to flows generated by elements of $\mathcal{A} C$ as gauge. In the classical case, the constraint $C$ and the function $f C$, where $f$ is any phase-space function not equal to zero on the constraint surface, generate the same gauge flow on the constraint surface: For $f C$, the flow equations

$$
\frac{\mathrm{d}}{\mathrm{d} \lambda^{\prime}}=\{\cdot f C\} \approx f\{\cdot, C\}
$$

can be mapped to the flow equations generated by $C$ by rescaling the gauge parameter $\lambda^{\prime}$ such that $\mathrm{d} \lambda=f \mathrm{~d} \lambda^{\prime}$. (For this reparameterization of the flow, the phase-space function $f$ is considered to be a function of $\lambda^{\prime}$ on the space of solutions of the flow equations. The sign " $\approx$ " in the preceding equations refers to equality on the constraint surface, as usual in the theory of constrained systems following Dirac.) In singly constrained quantum systems, by contrast, the gauge flows generated by $C$ and $A C$ with some $A \in \mathcal{A}$ are inequivalent: For $\omega \in \Gamma_{C}$, we have

$$
\omega([B, A C])=\omega(A[B, C]) \neq \omega(A) \omega([B, C])
$$

in general.

Remark: If zero is in the discrete spectrum of $C$ represented on the kinematical Hilbert space, the behavior of flows is rather different. Any quantum flow generated by $A C$ is then equivalent to the flow generated by $C$ when restricted to constrained pure states, given by vectors $\psi$ in the Hilbert space such that $C \psi=0$ : on these states, $S_{A C}(\lambda) \psi=$ $\exp (-i \lambda A C / \hbar) \psi$ such that

$$
S_{A C}(\lambda) \psi=\sum_{n=0}^{\infty} \frac{1}{n !}\left(\frac{-i \lambda}{\hbar}\right)^{n}(A C)^{n} \psi=0
$$

for any $A$. The full gauge flow may nevertheless be non-trivial on general algebraic states of Definition 3 .

Lemma 4 The constraint surface $\Gamma_{C}$ is preserved by the flow induced by any algebra element $A C$.

Proof: For any fixed $A \in \mathcal{A}$ and $\omega \in \Gamma_{C}$, following the same argument as in Lemma 2 , define functions $f_{B}(\lambda)=S_{A C}(\lambda) \omega(B C)$ on $\Gamma$, for $B \in \mathcal{A}$. Suppose all $f_{B}\left(\lambda^{\prime}\right)=0$ for some $\lambda^{\prime}$, then

$$
\begin{aligned}
\left.i \hbar \frac{\mathrm{d} f_{B}}{\mathrm{~d} \lambda}\right|_{\lambda=\lambda^{\prime}} & =\left.i \hbar \frac{\mathrm{d}}{\mathrm{d} \lambda}\left(S_{A C}(\lambda) \omega(B C)\right)\right|_{\lambda=\lambda^{\prime}} \\
& =\left.S_{A C}(\lambda) \omega([B C, A C])\right|_{\lambda=\lambda^{\prime}} \\
& =S_{A C}\left(\lambda^{\prime}\right) \omega(([B C, A]+A[B, C]) C)=f_{([B C, A]+A[B, C])}\left(\lambda^{\prime}\right)=0
\end{aligned}
$$


for all $B \in \mathcal{A}$. Moreover, we have initial conditions $f_{B}(0)=\omega(B C)=0$ for all $B$. It follows that $\left\{f_{B}(\lambda)=0, \forall \lambda\right\}_{B \in \mathcal{A}}$ is the solution to the flow induced by any algebra element of the form $A C$ that satisfies our initial conditions. Therefore, $S_{A C}(\lambda) \omega(B C)=0$ for all $\lambda$, and $S_{A C}(\lambda) \omega \in \Gamma_{C}$.

Any two $C$-equivalent states on $\Gamma_{C}$ are indistinguishble by their evaluation in Dirac observables:

Lemma 5 For any $\omega, \psi \in \Gamma_{C}$, if $\omega \sim_{C} \psi$, then $\omega(O)=\psi(O)$ for any $O \in \mathcal{A}_{\text {obs }}$.

Proof: The two states $\omega$ and $\psi$ are related by a succession of gauge flows $S_{A C}(\lambda)$. By Lemma 4, each of these flows preservers $\Gamma_{C}$. Therefore, for any $A \in \mathcal{A}$ and $B \in \mathcal{A}_{\text {obs }}$,

$$
\begin{aligned}
i \hbar \frac{\mathrm{d}}{\mathrm{d} \lambda}\left(S_{A C}(\lambda) \omega(B)\right) & =S_{A C}(\lambda) \omega([B, A C]) \\
& =S_{A C}(\lambda) \omega(A[B, C]+[B, A] C) \\
& =S_{A C}(\lambda) \omega([B, A] C)=0
\end{aligned}
$$

since $S_{A C}(\lambda) \omega \in \Gamma_{C}$. Therefore, $S_{A C}(\lambda) \omega(B)$ is constant along any gauge flow $S_{A C}(\lambda)$.

Equivalence classes $[\omega]_{C} \in \Gamma_{C} / \sim_{C}$ therefore define states on $\mathcal{A}_{\text {obs }}$.

Corollary 1 The space of physical states $\Gamma_{\text {phys }}$ is the convex subset of $\Gamma_{C} / \sim_{C}$ containing all $[\omega]_{C}$ with $\omega$ positive on $\mathcal{A}_{\text {obs }}$.

The computation of a complete $\mathcal{A}_{\text {obs }}$ is usually very complicated in interesting models. Moreover, a sufficiently large $\mathcal{A}_{\text {obs }}$ containing observables that can describe all measurements of interest may not exist, in particular in chaotic systems [30, 31]. The result of Corollary 1 partially avoids a direct reference to $\mathcal{A}_{\text {obs }}$ by formulating the space of physical states through an equivalence relation on the constrained states. However, one still needs access to $\mathcal{A}_{\text {obs }}$ in order to implement the positivity condition. This reference to $\mathcal{A}_{\text {obs }}$ cannot be avoided because it is generally impossible to extend positivity to all of $\mathcal{A}$ for any $\omega \in \Gamma_{C}$ : for any $A=A^{*} \in \mathcal{A}$ and a positive $\omega \in \Gamma$, we have $\omega(A C+C A) \in \mathbb{R}$ and $\omega([A, C]) \in i \mathbb{R}$. However for $\omega \in \Gamma_{C}$ we must have

$$
\omega(A C+C A)=\omega(2 A C-[A, C])=-\omega([A, C]),
$$

which, given the reality conditions, can be satisfied only if $\omega(A C+C A)=\omega([A, C])=0$. If $C$ possesses a canonical conjugate $Z=Z^{*} \in \mathcal{A}$ such that $[Z, C]=i \hbar \mathbf{1}$ (as we will shortly demand for a "clock"), then for any normalized state $\omega([Z, C])=i \hbar \neq 0$, so that no solution of the constraint is positive on all of $\mathcal{A}$. Moreover, as in the classical case, there is no evolution for physical states in a dynamically constrained system, since the adjoint action of the constraint has been factored out.

In Section 3 we will solve both problems - formulating positivity conditions without reference to $\mathcal{A}_{\text {obs }}$ and obtaining a consistent evolution picture — by introducing a new 
notion of gauge sections. Our approach is motivated by the algebraic analogue of deparameterization discussed in Section 2.1, In an unconstrained quantum system the dynamical flow is usually driven by some self-adjoint Hamiltonian $H=H^{*} \in \mathcal{B}$ via

$$
\frac{\mathrm{d} \omega_{t}(B)}{\mathrm{d} t}=\frac{1}{i \hbar} \omega_{t}([B, H])+\omega_{t}\left(\frac{\mathrm{d}}{\mathrm{d} t} B\right)
$$

where the derivation $\frac{1}{i \hbar}[\cdot, H]+\frac{\mathrm{d}}{\mathrm{d} t}(\cdot)$ clearly satisfies Definition 4 . The above evolution of states can be formulated as a pure adjoint action of a constraint by extending the kinematical algebra $\mathcal{B}$ by two new generators, "time" $Z=Z^{*}$ and "energy" $E=E^{*}$, such that $[Z, E]=i \hbar \mathbf{1}$ and $[Z, B]=0=[E, B]$ for all $B$ in $\mathcal{B}$. On this extended algebra $\mathcal{A}$, the constraint $C:=E+H \in \mathcal{A}$ generates a gauge flow

$$
\frac{\mathrm{d}}{\mathrm{d} \lambda} \omega_{\lambda}(A)=\frac{1}{i \hbar} \omega_{\lambda}([A, C])=\frac{1}{i \hbar} \omega_{\lambda}([A, H]+[A, E])=\frac{\omega_{\lambda}([A, H])}{i \hbar}+\omega_{\lambda}\left(\frac{\mathrm{d}}{\mathrm{d} Z} A\right)
$$

resembling the original dynamical flow on $\mathcal{B}$ for any $A$ polynomial in $Z$. Explicit time dependence of $B(t)$ in the Hamiltonian case corresponds to $Z$-dependence of $A \in \mathcal{A}$ in the constrained case. This process is called parameterization of the dynamical flow: physical time $t$ has been replaced by an arbitrary flow parameter $\lambda$.

One can follow this process in reverse: starting with the degrees of freedom described by the extended algebra $\mathcal{A}$ and given a Hamiltonian constraint one could attempt to deparameterize the system, reducing it to a an unconstrained dynamical system on a subalgebra $\mathcal{B} \subset \mathcal{A}$. In the above parameterized theory, the gauge flow (6]) is equivalent to the dynamical flow (5) if $Z \in \mathcal{A}$ can be "demoted to a real number" $t$. The deparameterization process of passing from $\mathcal{A}$ back to the smaller algebra $\mathcal{B}$ can therefore be interpreted as finding the states on $\mathcal{A}$ for which "the value of $Z$ is fixed" to equal $t$. For a general Hamiltonian constraint, this process requires finding a suitable clock $Z=Z^{*}$ : its values will keep track of time, and an associated algebra (the fashionables of Definition 2) will play the role of the smaller "unextended" algebra of evolving degrees of freedom.

\subsection{Quantum clocks}

Any kinematical observable $Z=Z^{*} \in \mathcal{A}$ can formally serve as a quantum reference system. In Section 3 we will use such reference systems to track translations in time, where $Z$ will serve as a "clock." (Reference systems can be used to track spatial translations as well [32, 33, 34, 35].) Following Lemma 3 the commutant $Z^{\prime}=\{A \in \mathcal{A}:[A, Z]=0\}$ is a unital *-subalgebra of $\mathcal{A}$. Furthermore, it is straightforward to verify that for any $t \in \mathbb{R}$ the set $(Z-t \mathbf{1}) Z^{\prime}$ is a two-sided $*$-ideal of $Z^{\prime}$. It follows that the quotient $Z^{\prime} /(Z-t \mathbf{1}) Z^{\prime}$ is unital and inherits a $*$-structure under the canonical projection $\pi_{t}: Z^{\prime} \rightarrow Z^{\prime} /(Z-t \mathbf{1}) Z^{\prime}$. In order to be useful for keeping track of time, such a quantum reference system needs some additional structure.

Definition 9 A quantum clock $(Z, \mathcal{F})$ is a reference observable $Z=Z^{*} \in \mathcal{A}$ together with a compatible fashionable algebra: a unital $*$-subalgebra $\mathcal{F} \subset Z^{\prime}$, such that for all $t \in \mathbb{R}$, we have $\mathcal{F} \cap$ ker $\pi_{t}=\{0\}$ and $\pi_{t}(\mathcal{F})=Z^{\prime} /(Z-t \mathbf{1}) Z^{\prime}$. 
Remark: Keeping with the commonsense physics usage, the term "clock" will also be used to refer to the reference observable $Z$ itself, where it will be assumed that $Z$ possesses a compatible fashionable algebra.

The two conditions on $\mathcal{F}$ guarantee that $\pi_{t}$ restricted to $\mathcal{F}$ is a $*$-algebra isomorphism. The algebra of fashionables is therefore a realization of a family of quotient algebras $Z^{\prime} /(Z-t 1) Z^{\prime}$ as a single subalgebra of $Z^{\prime}$ (and hence of $\mathcal{A}$ ). We denote the $*$-isomorphism $\nu_{t}: Z^{\prime} /(Z-t \mathbf{1}) Z^{\prime} \rightarrow \mathcal{F}$, where for any $X \in Z^{\prime} /(Z-t \mathbf{1}) Z^{\prime}$,

$$
\nu_{t}(X):=\pi_{t}^{-1}(X) \cap \mathcal{F}
$$

yields a single element of $\mathcal{F}$. This isomorphism inverts $\pi_{t}$ when the latter is restricted to $\mathcal{F}$, so that $\pi_{t} \circ \nu_{t}=$ id. As a direct consequence, we note

Lemma 6 For every $t \in \mathbb{R}, \mathcal{F}+(Z-t 1) Z^{\prime}=Z^{\prime}$.

Additionally, for each value of $t$ we have a projection from $Z^{\prime}$ to its subalgebra $\mathcal{F}$ via the composition of $*$-homomorphisms $\nu_{t} \circ \pi_{t}$.

Each quotient algebra $Z^{\prime} /(Z-t \mathbf{1}) Z^{\prime}$ possesses a natural relational interpretation:

Definition 10 For any kinematical observable $A=A^{*} \in \mathcal{A}$ that commutes with a clock $Z$, the relational observable for $A$ when the value of $Z$ is $t \in \mathbb{R}$ is the image of $A$ under the canonical projection, $A_{Z=t}:=\pi_{t}(A)$.

It is straightforward to check that $A_{Z=t}=A_{Z=t}^{*}$ under the inherited $*$-operation.

Remark: Time read by a reference clock behaves differently from the time of ordinary quantum mechanics where it is an external parameter. In the latter case any observable can be evaluated at a fixed time, in the former case this can only be done for observables that commute with the clock.

We will use the notation $\bar{\omega} \in \Gamma_{Z^{\prime}}$ to denote normalized states on $Z^{\prime}$. For each $t$, canonical projection $\pi_{t}$ defines a "surface of constant time" within $\Gamma_{Z^{\prime}}$, which will be used in Section 3.1 to fix the gauge flow of a quantum Hamiltonian constraint.

Definition 11 For any $t \in \mathbb{R}$ the surface of constant time $t$ of a clock $Z$ is

$$
\left.\Gamma_{Z^{\prime}}\right|_{\pi_{t}}=\left\{\bar{\omega} \in \Gamma_{Z^{\prime}}: \bar{\omega}(B)=0 \text { for all } B \in \operatorname{ker} \pi_{t}\right\} .
$$

Each state on this surface is a pull-back $\left.\Gamma_{Z^{\prime}}\right|_{\pi_{t}} \ni \bar{\omega}=\tilde{\omega} \circ \pi_{t}$ of a state $\tilde{\omega}$ on the quotient algebra $Z^{\prime} /(Z-t \mathbf{1}) Z^{\prime}$. The states belonging to such a surface that are positive on $Z^{\prime}$ can be interpreted as assigning a value to each $A=A^{*} \in Z^{\prime}$ "when clock $Z$ shows time $t^{\prime}$ ". Not every positive state $\bar{\omega}$ on the algebra $Z^{\prime}$ has a relational interpretation since this would require $\bar{\omega}((Z-t \mathbf{1}) A)=0$ for any $A \in Z^{\prime}$ for some $t \in \mathbb{R}$. A sequence of relational states provides a notion of evolution over time in clock $Z$. 
Definition 12 A one-parameter family of states $\bar{\omega}_{t} \in \Gamma_{Z^{\prime}}$ for $t \in(a, b) \subset \mathbb{R}$ is a time evolution of $\bar{\omega} \in \Gamma_{Z^{\prime}}$ relative to $Z$ if $\bar{\omega}=\bar{\omega}_{t_{0}}$ for some $t_{0} \in(a, b)$, and if for each $t \in(a, b)$ $\bar{\omega}_{t}$ is positive on $Z^{\prime}$ and $\left.\bar{\omega}_{t} \in \Gamma_{Z^{\prime}}\right|_{\pi_{t}}$.

While any positive state on a quotient $Z^{\prime} /(Z-t \mathbf{1}) Z^{\prime}$ does have a relational interpretation, these quotients give distinct algebras for different values of $t$. So a time evolution in $Z$ corresponds to a sequence of states on different disconnected algebras. The fashionable algebra is needed precisely to "sew together" constant time degrees of freedom at different values of the clock and to define clock-value-dependent states that can be freely specified over a fixed algebra $\mathcal{F}$. We define a one-parameter family of invertible linear maps $\psi_{t}:\left.\Gamma_{Z^{\prime}}\right|_{\pi_{t}} \rightarrow \Gamma_{\mathcal{F}}$ with the forward map given simply by restriction, for $\left.\bar{\omega} \in \Gamma_{Z^{\prime}}\right|_{\pi_{t}}$

$$
\psi_{t}(\bar{\omega})(F)=\bar{\omega}(F), \text { for all } F \in \mathcal{F} .
$$

We define its inverse using (7), for $\tilde{\omega} \in \Gamma_{\mathcal{F}}$

$$
\psi_{t}^{-1}(\tilde{\omega})(A)=\tilde{\omega}\left(\nu_{t}\left(\pi_{t}(A)\right)\right), \text { for all } A \in Z^{\prime} .
$$

Clearly, because it involves $\pi_{t}$, the resultant state $\left.\psi_{t}^{-1}(\tilde{\omega}) \in \Gamma_{Z^{\prime}}\right|_{\pi_{t}}$. It is also not difficult to verify that $\psi$ and $\psi^{-1}$ invert each other on their domains of definition. The fashionable algebra gives us the structure necessary to define time translation of a state from $Z=t_{1}$ to $Z=t_{2}:\left.\bar{\omega}_{1} \in \Gamma_{Z^{\prime}}\right|_{\pi_{t_{1}}}$ and $\left.\bar{\omega}_{2} \in \Gamma_{Z^{\prime}}\right|_{\pi_{t_{2}}}$ represent the same unevolved state at two different times $t_{1}$ and $t_{2}$ if $\bar{\omega}_{1}(F)=\bar{\omega}_{2}(F)$ for all $F \in \mathcal{F}$, that is, if $\bar{\omega}_{1}=\psi_{t_{1}}^{-1}(\tilde{\omega})$ and $\bar{\omega}_{2}=\psi_{t_{2}}^{-1}(\tilde{\omega})$ for some $\tilde{\omega} \in \Gamma_{\mathcal{F}}$.

A time evolution maps to a one-parameter family of positive states on the fashionable algebra via $\tilde{\omega}_{t}=\psi_{t}\left(\bar{\omega}_{t}\right)$. Conversely, any one-parameter family of positive states $\tilde{\omega}_{t} \in \Gamma_{\mathcal{F}}$ maps to a time evolution with respect to $Z$ via $\bar{\omega}_{t}=\psi_{t}^{-1}\left(\tilde{\omega}_{t}\right)$. The maps $\psi_{t}$ and $\psi_{t}^{-1}$ preserve positivity of states, because the underlying algebra maps that they utilize, $\imath: \mathcal{F} \hookrightarrow Z^{\prime}, \pi_{t}$, and $\nu_{t}$ are all $*$-homomorphisms. This can be stated somewhat more broadly.

Lemma 7 If $\left.\bar{\omega} \in \Gamma_{Z^{\prime}}\right|_{\pi_{t}}$ for some $t \in \mathbb{R}$ and $\bar{\omega}$ is positive on $\mathcal{F} \subset Z^{\prime}$, then $\bar{\omega}$ is positive on the whole of $Z^{\prime}$.

Proof: Since $\left.\bar{\omega} \in \Gamma_{Z^{\prime}}\right|_{\pi_{t}}$, we have $\bar{\omega}((Z-t \mathbf{1}) A)=0$ for all $A \in Z^{\prime}$. According to Lemma 6 , for any $B \in Z^{\prime}$ there are $F \in \mathcal{F} \subset Z^{\prime}$ and $B_{0} \in Z^{\prime}$ such that $B=F+(Z-t 1) B_{0}$. Thus

$$
\begin{aligned}
\bar{\omega}\left(B B^{*}\right) & =\bar{\omega}\left(F F^{*}+F(Z-t \mathbf{1}) B_{0}^{*}+(Z-t \mathbf{1}) B_{0} F^{*}+(Z-t \mathbf{1}) B_{0}(Z-t \mathbf{1}) B_{0}^{*}\right) \\
& =\bar{\omega}\left(F F^{*}\right)+\bar{\omega}\left((Z-t \mathbf{1})\left(F B_{0}^{*}+B_{0} F^{*}+(Z-t \mathbf{1}) B_{0} B_{0}^{*}\right)\right) \\
& =\bar{\omega}\left(F F^{*}\right) \geq 0 .
\end{aligned}
$$

In what follows we use a clock as the basis for a new method of deparameterization by (i) using the commutant $Z^{\prime} \subset \mathcal{A}$ as a go-between of the kinematical algebra $\mathcal{A}$ and the algebra 
of observables, $\mathcal{A}_{\text {obs }}$, (ii) fixing gauge degrees of freedom using constant time surfaces $\left.\Gamma_{Z^{\prime}}\right|_{\pi_{t}}$, and (iii) demoting the clock observable $Z$ to a real-valued time parameter $t$. The constanttime surfaces are analogous to fixing an initial $\lambda$-time in standard deparameterization discussed in Section 2.1, but they are introduced in a subalgebra of $\mathcal{A}$ and do not require the transition to an unrelated space such as $\mathcal{H}_{\text {phys }}$. Moreover, Dirac observables will be replaced by the more accessible fashionables $\mathcal{F}$. (Since their definition depends on the choice of time $Z$, and not just on the constraint $C$, they are "in fashion" only as long as $Z$ is used as time. This need to introduce fashionables has first been identified in semiclassical calculations [27, 28, 29].)

\section{Quantum dynamical reduction: Linear case}

In this section, we assume that the constraint is of a form $C=C_{H}$ such that $\left[Z, C_{H}\right]=i \hbar \mathbf{1}$ for some $Z \in \mathcal{A}$. This case is close to standard deparameterization, as shown by (66). (As we will discuss in more detail in Section 4, a constraint should, in general, be factorized in order to make it deparameterizable, in the sense that $C=N C_{H}$ with suitable $C_{H}=C_{H}^{*}$, $N \in \mathcal{A}$.)

Definition 13 A quantum constraint $C_{H} \in \mathcal{A}$ is deparameterized by the clock $(Z, \mathcal{F})$ if $\left[Z, C_{H}\right]=i \hbar \mathbf{1}$ and the commutant of $Z$ has the following properties:

1. $Z^{\prime}+\mathcal{A} C_{H}=\mathcal{A}$;

2. $Z^{\prime} \cap \mathcal{A} C_{H}=\{0\}$;

3. the set $Z^{\prime} \cup\left\{C_{H}\right\}$ algebraically generates $\mathcal{A}$.

The main result of this section is that deparameterization of a constraint $C_{H}$ by a clock $(Z, \mathcal{F})$ is a realization of the constrained quantization of $C_{H}$ as an unconstrained quantum mechanical system with degrees of freedom given by the fashionable algebra $\mathcal{F}$ evolving in time. The consistency of this construction is shown by

Proposition 2 If $C_{H} \in \mathcal{A}$ is deparameterized by $(Z, \mathcal{F})$, then there is a bijection between the constraint surface and the space of states on $Z^{\prime}, \phi: \Gamma_{C_{H}} \rightarrow \Gamma_{Z^{\prime}}$ such that:

1. All gauge flows mapped to $\Gamma_{Z^{\prime}}$ by $\phi$ are transversal to the constant time surfaces $\left.\Gamma_{Z^{\prime}}\right|_{\pi_{t}}, t \in \mathbb{R}$.

2. The flow generated by $C_{H}$ itself is mapped to a dynamical flow on $\mathcal{F}$.

3. Each positive state on $\mathcal{F}$ specifies a positive physical state $[\omega]_{C_{H}} \in \Gamma_{C_{H}} / \sim_{C_{H}}$.

Existence of $\phi$ follows immediately from conditions 1 and 2 of Definition 13 . Since $Z^{\prime}+\mathcal{A} C_{H}=\mathcal{A}$, any linear functional on $\mathcal{A}$ is completely defined by its restrictions to $\mathcal{A} C_{H}$ and $Z^{\prime}$. Since $Z^{\prime} \cap \mathcal{A} C_{H}=\{0\}$ these two restrictions can be specified independently. Any 
state in $\Gamma_{C_{H}}$ vanishes on $\mathcal{A} C_{H}$ and defines a state on $\Gamma_{Z^{\prime}}$ by restriction to $Z^{\prime}$. Conversely any state on $Z^{\prime}$ can be uniquely extended to the entirety of $\mathcal{A}$ by setting the extension to vanish on $\mathcal{A} C_{H}$ (see equations (11) and (12) below). Deparameterization provides sections of the quantum gauge flow (Definition 8) through constant-time surfaces $\left.\Gamma_{Z^{\prime}}\right|_{\pi_{t}}$. Transversality of these sections, defined and proven in Section 3.1, is the local quantum analog of incisiveness of a classical gauge section of Definition 1. Potential issues arising from the weaker local nature of transversality are discussed in Section 3.5. We prove properties 2 and 3 of the proposition in Sections 3.2 and 3.3. respectively, and provide an explicit example of a deparameterizable constraint in Section 3.4 .

Remark: As shown in our discussion of cosmological models, condition 3 of Definition 13 is satisfied by the algebras of many physical examples of interest where $C_{H}$ and kinematical observables are polynomial in an "energy" $E \in \mathcal{A}$. This property is only used in Section 3.1 specifically to ensure that all gauge flow generators can be projected to $Z^{\prime}$ using finite power series. In other situations it may be possible to replace this condition with sufficiently strong assumptions on the topology of $\mathcal{A}$.

\subsection{Proof of transversality}

Let a quantum constraint $C_{H}$ be deparameterized by a clock $(Z, \mathcal{F})$. In this subsection we prove that all of the flows generated by constraint elements $A C_{H} \in \mathcal{A} C_{H}$ can be mapped to $\Gamma_{Z^{\prime}}$, and are separated by the constant-time surfaces of $Z$. According to Definition 11, a constant-time surface $\left.\Gamma_{Z^{\prime}}\right|_{\pi_{t}}$ in $\Gamma_{Z^{\prime}}$ contains precisely those states on $Z^{\prime}$ that vanish on ker $\pi_{t}$. A non-vanishing gauge flow that preserves the values assigned by states to ker $\pi_{t}$ is therefore tangent to $\left.\Gamma_{Z^{\prime}}\right|_{\pi_{t}}$ and hence remains unresolved by fixing the value of the clock. By contrast, a flow that is separated by the $\left.\Gamma_{Z^{\prime}}\right|_{\pi_{t}}$ either pierces the surface or vanishes at each of its points. The corresponding geometrical picture suggests a notion of transversality.

Definition 14 1. A one-parameter family of states $\bar{\omega}_{\lambda} \in \Gamma_{Z^{\prime}}$ is transversal to a constant time surface $\left.\Gamma_{Z^{\prime}}\right|_{\pi_{t}}$ if for all $\lambda^{\prime}$ such that $\left.\bar{\omega}_{\lambda^{\prime}} \in \Gamma_{Z^{\prime}}\right|_{\pi_{t}}$ either $\left.\frac{\mathrm{d}}{\mathrm{d} \lambda} \bar{\omega}_{\lambda}(A)\right|_{\lambda=\lambda^{\prime}}=$ $0, \forall A \in Z^{\prime}$ or there is some $B \in \operatorname{ker} \pi_{t}$ such that $\left.\frac{\mathrm{d}}{\mathrm{d} \lambda} \bar{\omega}_{\lambda}(B)\right|_{\lambda=\lambda^{\prime}} \neq 0$.

2. A flow on $Z^{\prime}$ is transversal to $\left.\Gamma_{Z^{\prime}}\right|_{\pi_{t}}$ if every one-parameter family of states generated by it is transversal to $\left.\Gamma_{Z^{\prime}}\right|_{\pi_{t}}$.

To map gauge flows onto $\Gamma_{Z^{\prime}}$, let us first explicitly define the bijection between $\Gamma_{C_{H}}$ and $\Gamma_{Z^{\prime}}$. Taken together, the two conditions of deparameterization $Z^{\prime} \cap \mathcal{A} C_{H}=\{0\}$ and $Z^{\prime}+\mathcal{A} C_{H}=\mathcal{A}$ imply that every $A$ can be written as

$$
A=B+G C_{H}
$$

where $B \in Z^{\prime}$ is unique and $G \in \mathcal{A}$ is unique up to adding terms that are annihilated by $C_{H}$ multiplied on their right because $C_{H}$ is not a divisor of zero in a single constrained 
system. We define the forward map $\phi: \Gamma_{C_{H}} \rightarrow \Gamma_{Z^{\prime}}$ by restriction: for any $\omega \in \Gamma_{C_{H}}$ and $A \in Z^{\prime}$

$$
\phi(\omega)(A)=\omega(A) .
$$

We use decomposition (10) to define the inverse map: for any $A \in \mathcal{A}$ and $\bar{\omega} \in \Gamma_{Z^{\prime}}$, we have $A=B+G C_{H}$, and define

$$
\phi^{-1}(\bar{\omega})(A)=\bar{\omega}(B) .
$$

Since $B=0$ for any $A \in \mathcal{A} C_{H}$ by condition 2 of Definition 13 , clearly $\phi^{-1}(\bar{\omega}) \in \Gamma_{C_{H}} \subset \Gamma$. A flow is mapped from $\Gamma_{C_{H}}$ to $\Gamma_{Z^{\prime}}$ by mapping the one-parameter families of states it generates: $\bar{\omega}_{\lambda}=\phi\left(\omega_{\lambda}\right)$.

We can further iterate relation (10) expressing $G$ as a sum of elements from $Z^{\prime}$ and $\mathcal{A} C_{H}$ to get a second-order expression $A=B+\tilde{B} C_{H}+\tilde{G} C_{H}^{2}$ with $B, \tilde{B} \in Z^{\prime}$ and $\tilde{G} \in \mathcal{A}$, and so on. In fact, since by condition 3 of Definition 13 the set $Z^{\prime} \cup\left\{C_{H}\right\}$ algebraically generates $\mathcal{A}$, every $A \in \mathcal{A}$ can be written as a finite-order polynomial in $C_{H}$

$$
A=B_{0}+B_{1} C_{H}+B_{2} C_{H}^{2}+\ldots B_{M} C_{H}^{M}
$$

with $B_{i} \in Z^{\prime}$ and $M \in \mathbb{N}$.

Further, the adjoint action of $C_{H}$ preserves the commutant of $Z$, since for any $A \in Z^{\prime}$

$$
\left[\left[A, C_{H}\right], Z\right]=\left[\left[Z, C_{H}\right], A\right]+\left[[A, Z], C_{H}\right]=0
$$

since $\left[Z, C_{H}\right]=i \hbar \mathbf{1}$ according to Definition $\left[13\right.$, and $[A, Z]=0$; therefore $\left[A, C_{H}\right] \in Z^{\prime}$.

Lemma 8 For a constraint $C_{H}$ deparameterized by a clock $(Z, \mathcal{F})$, every gauge flow mapped to $\Gamma_{Z^{\prime}}$ from $\Gamma_{C_{H}}$ is determined by the derivation $\vec{D}_{H} F=\frac{1}{i \hbar}\left[F, C_{H}\right]$ on $Z^{\prime}$.

Proof: First, we note that for $F, B \in \mathcal{A}$

$$
\left[F, B C_{H}\right]=B\left(i \hbar \vec{D}_{H} F\right)+[F, B] C_{H}
$$

Iterating by replacing $B$ with $B C_{H}$, we get for any integer $n \geq 1$,

$\left[F, B C_{H}^{n}\right]=\sum_{i=1}^{n}\left(\begin{array}{c}n \\ i\end{array}\right)(-1)^{i-1} B\left((i \hbar)^{i} \vec{D}_{H}^{i} F\right) C_{H}^{n-i}+[F, B] C_{H}^{n}=(-1)^{n-1} B\left((i \hbar)^{n} \vec{D}_{H}^{n} F\right)+G C_{H}$.

We have combined the terms proportional to $C_{H}$ using

$$
G=\sum_{i=1}^{n-1}\left(\begin{array}{l}
n \\
i
\end{array}\right)(-1)^{i-1} B\left((i \hbar)^{i} \vec{D}_{H}^{i} F\right) C_{H}^{n-i-1}+[F, B] C_{H}^{n-1}
$$

They all vanish when evaluated on states in $\Gamma_{C_{H}}$. Using the above result and writing $A \in \mathcal{A}$ as a polynomial in $C_{H}$ as in equation (13), we have

$$
\left[F, A C_{H}\right]=\sum_{n=1}^{M+1}(-1)^{n-1} B_{n-1}\left((i \hbar)^{n} \vec{D}_{H}^{n} F\right)+G C_{H}
$$


for some $G \in \mathcal{A}$ and $B_{i} \in Z^{\prime}$. For any state $\omega \in \Gamma_{C_{H}}$, we have $\omega\left(G C_{H}\right)=0$, and hence the flows induced by constraint elements $A C_{H}$ satisfy

$$
i \hbar \frac{\mathrm{d}}{\mathrm{d} \lambda} \omega_{\lambda}(F)=\omega_{\lambda}\left(\left[F, A C_{H}\right]\right)=\sum_{n=1}^{M+1}(-1)^{n-1} \omega_{\lambda}\left(B_{n-1}\left((i \hbar)^{n} \vec{D}_{H}^{n} F\right)\right)
$$

For $F \in Z^{\prime}$ we also have $\vec{D}_{H}^{n} F \in Z^{\prime}$, and the above expression can be computed entirely through the states' restriction to $Z^{\prime}$, and the action of $\vec{D}_{H}$ on $Z^{\prime}$. Hence, for $\bar{\omega}_{\lambda}=\phi\left(\omega_{\lambda}\right)$

$$
i \hbar \frac{\mathrm{d}}{\mathrm{d} \lambda} \bar{\omega}_{\lambda}(F)=\bar{\omega}_{\lambda}\left(\sum_{n=1}^{M+1}(-1)^{n-1}(i \hbar)^{n} B_{n-1} \vec{D}_{H}^{n}(F)\right) .
$$

For the flow generated on $\Gamma_{Z^{\prime}}$ by $C_{H}$ itself, $B_{0}=\mathbf{1}$ and $B_{n}=0$ for $n>1$ in (15). For $F=\mathbf{1}$ equation (16) implies that along the flow, $\mathrm{d} \bar{\omega}_{\lambda}((Z-t \mathbf{1})) / \mathrm{d} \lambda=1 \neq 0$ for any $t \in \mathbb{R}$. Therefore this particular flow is transversal to all constant time surfaces $\left.\Gamma_{Z^{\prime}}\right|_{\pi_{t}}$. In fact, the property

$$
\bar{\omega}\left(\left(\vec{D}_{H} Z\right) F\right)=\bar{\omega}(\mathbf{1} F)=\bar{\omega}(F)
$$

is sufficient to ensure that all gauge flows mapped to $\Gamma_{Z^{\prime}}$ are transversal to constant time surfaces. To show this, we first establish a useful result that holds for any $\vec{D}_{H} Z \in Z^{\prime}$ that commutes with all elements of $Z^{\prime}$, and a pair of non-negative integers $i \leq n$ :

$$
\begin{aligned}
\vec{D}_{H}^{i}(Z-t \mathbf{1})^{n} & =\vec{D}_{H}^{i-1}\left(n(Z-t \mathbf{1})^{n-1}\left(\vec{D}_{H} Z\right)\right) \\
& =\vec{D}_{H}^{i-2}\left(n(n-1)(Z-t \mathbf{1})^{n-2}\left(\vec{D}_{H} Z\right)^{2}+n(Z-t \mathbf{1})^{n-1}\left(\vec{D}_{H}^{2} Z\right)\right) \\
& =\cdots \\
& =(Z-t \mathbf{1})^{n-i}\left(\begin{array}{c}
n \\
i
\end{array}\right)\left(\vec{D}_{H} Z\right)^{i}+(Z-t \mathbf{1})^{n-i+1}(\cdots) .
\end{aligned}
$$

Therefore, for $\left.\bar{\omega} \in \Gamma_{Z^{\prime}}\right|_{\pi_{t}}$, and for any $F_{1}, F_{2} \in Z^{\prime}$

$$
\bar{\omega}\left[F_{1}\left(\vec{D}_{H}^{i}(Z-t \mathbf{1})^{n}\right) F_{2}\right]=\left\{\begin{array}{cl}
0 & \text { for } i<n ; \\
\bar{\omega}\left(\left(\vec{D}_{H} Z\right)^{n} F_{1} F_{2}\right) & \text { for } i=n .
\end{array}\right.
$$

Lemma 9 If $C_{H}$ is deparameterized by $(Z, \mathcal{F})$, the flow of every $A C_{H} \in \mathcal{A} C_{H}$ mapped to $\Gamma_{Z^{\prime}}$ is transversal to every constant time surface $\left.\Gamma_{Z^{\prime}}\right|_{\pi_{t}}$ of $Z$.

Proof: Using (13) we write

$$
A=B_{0}+B_{1} C_{H}+B_{2} C_{H}^{2}+\ldots B_{M} C_{H}^{M}
$$

with $B_{i} \in Z^{\prime}$. Suppose this flow is not transversal to $\left.\Gamma_{Z^{\prime}}\right|_{\pi_{t}}$ for some $t \in \mathbb{R}$. Then, according to Definition [14, there is a one-parameter family of states $\bar{\omega}_{\lambda} \in \Gamma_{Z^{\prime}}$ generated 
by $A C_{H}$ according to equation (16) with $\left.\bar{\omega}_{\lambda_{0}} \in \Gamma_{Z^{\prime}}\right|_{\pi_{t}}$ for some $\lambda_{0} \in \mathbb{R}$, such that the flow of $A C_{H}$ does not vanish at $\bar{\omega}_{\lambda_{0}}$, and for any $F \in Z^{\prime}$ using (16),

$$
\begin{aligned}
0 & =\left.i \hbar \frac{\mathrm{d}}{\mathrm{d} \lambda} \bar{\omega}_{\lambda}\left((Z-t \mathbf{1})^{M+1} F\right)\right|_{\lambda=\lambda_{0}} \\
& =\bar{\omega}_{\lambda_{0}}\left(\sum_{n=1}^{M+1}(-1)^{n-1}(i \hbar)^{n} B_{n-1} \vec{D}_{H}^{n}\left((Z-t \mathbf{1})^{M+1} F\right)\right) \\
& =\sum_{n=1}^{M+1}(-1)^{n-1}(i \hbar)^{n} \bar{\omega}_{\lambda_{0}}\left(B_{n-1} \sum_{m=1}^{n}\left(\begin{array}{c}
n \\
m
\end{array}\right)\left(\vec{D}_{H}^{m}(Z-t \mathbf{1})^{M+1}\right)\left(\vec{D}_{H}^{n-m} F\right)\right) \\
& =\sum_{n=1}^{M+1}(-1)^{n-1}(i \hbar)^{n} \sum_{m=1}^{n}\left(\begin{array}{c}
n \\
m
\end{array}\right) \bar{\omega}_{\lambda_{0}}\left(B_{n-1}\left(\vec{D}_{H}^{m}(Z-t \mathbf{1})^{M+1}\right)\left(\vec{D}_{H}^{n-m} F\right)\right) \\
& =\sum_{n=1}^{M+1}(-1)^{n-1}(i \hbar)^{n} \sum_{m=1}^{n}\left(\begin{array}{c}
n \\
m
\end{array}\right) \delta_{m, M+1} \bar{\omega}_{\lambda_{0}}\left(B_{n-1}\left(\vec{D}_{H} Z\right)^{M+1}\left(\vec{D}_{H}^{n-m} F\right)\right) \\
& =(-1)^{M}(i \hbar)^{M+1} \bar{\omega}_{\lambda_{0}}\left(B_{M} \mathbf{1}^{M+1} F\right)=(-1)^{M}(i \hbar)^{M+1} \bar{\omega}_{\lambda_{0}}\left(B_{M} F\right),
\end{aligned}
$$

where the Kronecker delta comes directly from (18). This implies that $\bar{\omega}_{\lambda_{0}}\left(B_{M} F\right)=0$ for all $F \in Z^{\prime}$. Iterating the argument, (16) also implies

$$
\begin{aligned}
0= & \left.i \hbar \frac{\mathrm{d}}{\mathrm{d} \lambda} \bar{\omega}_{\lambda}\left((Z-t \mathbf{1})^{M} F\right)\right|_{\lambda=\lambda_{0}} \\
= & \bar{\omega}_{\lambda_{0}}\left(\sum_{n=1}^{M+1}(-1)^{n-1}(i \hbar)^{n} B_{n-1} \vec{D}_{H}^{n}\left((Z-t \mathbf{1})^{M} F\right)\right) \\
= & \bar{\omega}_{\lambda_{0}}\left(\sum_{n=1}^{M}(-1)^{n-1}(i \hbar)^{n} B_{n-1} \vec{D}_{H}^{n}\left((Z-t \mathbf{1})^{M} F\right)\right) \\
& +(-1)^{M}(i \hbar)^{M+1} \bar{\omega}_{\lambda_{0}}\left(B_{M}\left[\vec{D}_{H}^{M+1}\left((Z-t \mathbf{1})^{M} F\right)\right]\right) .
\end{aligned}
$$

By (19), the second term in the final expression is zero for any $F \in Z^{\prime}$, giving

$$
\begin{aligned}
0 & =\bar{\omega}_{\lambda_{0}}\left(\sum_{n=1}^{M}(-1)^{n-1}(i \hbar)^{n} B_{n-1} \vec{D}_{H}^{n}\left((Z-t \mathbf{1})^{M} F\right)\right) \\
& =\sum_{n=1}^{M}(-1)^{n-1}(i \hbar)^{n} \sum_{m=1}^{n}\left(\begin{array}{c}
n \\
m
\end{array}\right) \bar{\omega}_{\lambda_{0}}\left(B_{n-1}\left(\vec{D}_{H}^{m}(Z-t \mathbf{1})^{M}\right)\left(\vec{D}_{H}^{n-m} F\right)\right) \\
& =(-1)^{M-1}(i \hbar)^{M} \bar{\omega}_{\lambda_{0}}\left(B_{M-1}\left(\vec{D}_{H} Z\right)^{M} F\right)=(-1)^{M-1}(i \hbar)^{M} \bar{\omega}_{\lambda_{0}}\left(B_{M-1} F\right)
\end{aligned}
$$

which implies $\bar{\omega}_{\lambda_{0}}\left(B_{M-1} F\right)=0$ for all $F \in Z^{\prime}$. Continuing in this way, we establish that $\bar{\omega}_{\lambda_{0}}\left(B_{n} F\right)=0$ for all $n$. Therefore the flow must completely vanish at $\bar{\omega}_{\lambda_{0}}$ since

$$
\left.i \hbar \frac{\mathrm{d}}{\mathrm{d} \lambda} \bar{\omega}_{\lambda}(F)\right|_{\lambda=\lambda_{0}}=\sum_{n=1}^{M+1}(-1)^{n-1}(i \hbar)^{n} \bar{\omega}\left(B_{n-1}\left(\vec{D}_{H}^{n} F\right)\right)=0
$$


proving our claim by contradiction.

\subsection{Deparameterized time evolution}

In this subsection we prove that for a constraint $C_{H}$ that is deparameterized by $(Z, \mathcal{F})$, the adjoint action of $C_{H}$ projects to a dynamical flow on the fashionable algebra $\mathcal{F}$. Later on, in Section 3.3 we will link deparameterized dynamics to the physical states and the Dirac observables of the constrained system.

Let $S_{C_{H}}(\lambda)$ denote the flow induced on $\Gamma$ by the adjoint action of $C_{H}$ on $\mathcal{A}$, which by Lemma 4 preserves the constraint surface $\Gamma_{C_{H}}$. Using the map defined in (11) and (12) we can transfer this flow from $\Gamma_{C}$ to a flow on $\Gamma_{Z^{\prime}}$ via $\bar{S}_{C_{H}}(\lambda)=\phi \circ S_{C_{H}}(\lambda) \circ \phi^{-1}$. Explicitly, $\bar{S}_{C_{H}}(\lambda) \phi(\omega)=\phi\left(S_{C_{H}}(\lambda) \omega\right)$ for any $\omega \in \Gamma_{C_{H}}$. Using this relation, for any $\bar{\omega} \in \Gamma_{Z^{\prime}}$ and any $A \in Z^{\prime}$

$$
i \hbar \frac{\mathrm{d}}{\mathrm{d} \lambda}\left(\bar{S}_{C_{H}}(\lambda) \bar{\omega}(A)\right)=\bar{S}_{C_{H}}(\lambda) \bar{\omega}\left(\left[A, C_{H}\right]\right),
$$

where we implicitly use the fact that $\left[A, C_{H}\right] \in Z^{\prime}$, which follows from equation (14). Therefore, $\bar{S}_{C_{H}}(\lambda)$ is generated simply by the adjoint action of $C_{H}$ on the subalgebra $Z^{\prime}$. Referring back to Definition 12 from Section 2.4 we have the following result.

Lemma 10 Let $C_{H}$ be deparameterized by $(Z, \mathcal{F})$, then the flow $\bar{S}_{C_{H}}(\lambda)=\phi \circ S_{C_{H}}(\lambda) \circ \phi^{-1}$ generates time evolution of states relative to $Z$ on $\Gamma_{Z^{\prime}}$.

Proof: Since $\left[\cdot, C_{H}\right]$ is a $*$-compatible derivation on $\mathcal{A}$, it is $*$-compatible also on $Z^{\prime}$. All that needs to be shown is that the gauge flow $\bar{S}_{C_{H}}(\lambda)$ maps states from $\left.\Gamma_{Z^{\prime}}\right|_{\pi_{t}}$ to $\left.\Gamma_{Z^{\prime}}\right|_{\pi_{t+\lambda}}$.

For convenience let us denote the one-parameter family of states $\bar{\omega}_{\lambda}:=\bar{S}_{C_{H}}(\lambda) \bar{\omega}$, where $\left.\bar{\omega} \in \Gamma_{Z^{\prime}}\right|_{\pi_{t}}$ for some fixed $t$. Following the method of Lemma 2 , for each $A \in Z^{\prime}$ we define a function that varies along the flow $f_{A}(\lambda)=\bar{\omega}_{\lambda}((Z-(t+\lambda) \mathbf{1}) A)$. The state $\bar{\omega}_{\lambda}$ belongs to $\left.\Gamma_{Z^{\prime}}\right|_{\pi_{t+\lambda}}$ if and only if $f_{A}(\lambda)=\bar{\omega}_{\lambda}((Z-(t+\lambda) \mathbf{1}) A)=0$ for all $A \in Z^{\prime}$. Suppose all of the functions $f_{A}\left(\lambda^{\prime}\right)=0$ for some $\lambda^{\prime}$. We can compute their derivatives along the flow using equation (20):

$$
\begin{aligned}
\frac{\left.\mathrm{d} f_{A}\right|_{\lambda=\lambda^{\prime}}}{\mathrm{d} \lambda} & =\left.\frac{\mathrm{d}}{\mathrm{d} \lambda} \bar{\omega}_{\lambda}((Z-(t+\lambda) \mathbf{1}) A)\right|_{\lambda=\lambda^{\prime}} \\
& =\left.\frac{\mathrm{d}}{\mathrm{d} \lambda}\left(\bar{\omega}_{\lambda}((Z-t \mathbf{1}) A)-\lambda \bar{\omega}_{\lambda}(A)\right)\right|_{\lambda=\lambda^{\prime}} \\
& =\frac{1}{i \hbar} \bar{\omega}_{\lambda^{\prime}}\left(\left[(Z-t \mathbf{1}) A, C_{H}\right]\right)-\frac{\lambda^{\prime}}{i \hbar} \bar{\omega}_{\lambda^{\prime}}\left(\left[A, C_{H}\right]\right)-\bar{\omega}_{\lambda^{\prime}}(A) \\
& =\frac{1}{i \hbar} \bar{\omega}_{\lambda^{\prime}}\left((Z-t \mathbf{1})\left[A, C_{H}\right]\right)-\frac{\lambda^{\prime}}{i \hbar} \bar{\omega}_{\lambda^{\prime}}\left(\left[A, C_{H}\right]\right) \\
& =\frac{1}{i \hbar} \bar{\omega}_{\lambda^{\prime}}\left(\left(Z-\left(t+\lambda^{\prime}\right) \mathbf{1}\right)\left[A, C_{H}\right]\right)=\frac{1}{i \hbar} f_{\left[A, C_{H}\right]}\left(\lambda^{\prime}\right)=0 .
\end{aligned}
$$


The last equality follows since $\left[A, C_{H}\right] \in Z^{\prime}$. Furthermore, by our initial conditions $f_{A}(0)=$ 0 for all $A \in \mathcal{A}$ since $\bar{\omega}_{0}=\left.\bar{\omega} \in \Gamma_{Z^{\prime}}\right|_{\pi_{t+0}}$. It follows that $\left\{f_{A}(\lambda)=0 \text {, for all } \lambda\right\}_{A \in \mathcal{A}}$ is the solution to the flow given by equation (20) with $\left.\bar{\omega} \in \Gamma_{Z^{\prime}}\right|_{\pi_{t}}$. Thus at any point along the flow

$$
\bar{\omega}_{\lambda}((Z-(t+\lambda) \mathbf{1}) A)=0, \text { for all } A \in Z^{\prime} .
$$

Therefore $\left.\bar{S}_{C_{H}}(\lambda) \bar{\omega} \in \Gamma_{Z^{\prime}}\right|_{\pi_{t+\lambda}}$, as required.

Remark: The above lemma does not guarantee that for an arbitrary $\omega \in \Gamma_{C_{H}}$ the family $\phi\left(S_{C_{H}}(\lambda) \omega\right)$ is a time evolution relative to $Z$; we also need $\left.\phi(\omega) \in \Gamma_{Z^{\prime}}\right|_{\pi_{t}}$ for some $t \in \mathbb{R}$.

As discussed in Section [2.4, time evolution of states $\bar{\omega}_{t} \in \Gamma_{Z^{\prime}}$ relative to $Z$ maps to a one-parameter family of states on the fashionable algebra $\tilde{\omega}_{t}=\psi_{t}\left(\bar{\omega}_{t}\right)$, by restriction of $\bar{\omega}_{t}$ to $\mathcal{F}$ : we have $\tilde{\omega}_{t}(F)=\bar{\omega}_{t}(F)$ for all $F \in \mathcal{F}$. Furthermore, at each value $t \in \mathbb{R}$ of the clock the adjoint action of $C_{H}$ can be projected from $Z^{\prime}$ to $\mathcal{F}$ using the map of equation (7)

$$
\vec{D}_{H}^{\prime}(t) F:=\nu_{t} \circ \pi_{t}\left(\vec{D}_{H} F\right),
$$

which is a $*$-compatible derivation on $\mathcal{F}$ thanks to the fact that $\nu_{t}$ and $\pi_{t}$ are $*$-homomorphisms. $\vec{D}_{H}^{\prime}(t)$ is therefore a dynamical flow on $\mathcal{F}$ according to Definition 4 .

Lemma 11 Let $\bar{\omega}_{t}=\bar{S}_{C_{H}}\left(t-t_{0}\right) \bar{\omega}$ for some $t_{0} \in \mathbb{R}$ and $\left.\bar{\omega} \in \Gamma_{Z^{\prime}}\right|_{\pi_{t_{0}}}$, then the image $\tilde{\omega}_{t}=\psi_{t}\left(\bar{\omega}_{t}\right) \in \Gamma_{\mathcal{F}}$, where $\psi_{t}$ is defined in (8) is generated by the dynamical flow $\vec{D}_{H}^{\prime}(t)$. That is

$$
\frac{\mathrm{d}}{\mathrm{d} t} \tilde{\omega}_{t}(F)=\tilde{\omega}_{t}\left(\vec{D}_{H}^{\prime}(t) F\right)
$$

Proof: By Lemma 10, $\left.\bar{\omega}_{t} \in \Gamma_{Z^{\prime}}\right|_{\pi_{t}}$ for all $t$. Using equation (20), for any $A \in Z^{\prime}$

$$
\frac{\mathrm{d}}{\mathrm{d} t} \bar{\omega}_{t}(A)=\bar{\omega}_{t}\left(\frac{1}{i \hbar}\left[A, C_{H}\right]\right)=\bar{\omega}_{t}\left(\vec{D}_{H} F\right) .
$$

Since $\tilde{\omega}_{t}(F)=\psi_{t}\left(\bar{\omega}_{t}\right)(F)=\bar{\omega}_{t}(F)$ for any $F \in \mathcal{F}$, we also have

$$
\frac{\mathrm{d}}{\mathrm{d} t} \tilde{\omega}_{t}(F)=\frac{\mathrm{d}}{\mathrm{d} t} \bar{\omega}_{t}(F)=\bar{\omega}_{t}\left(\vec{D}_{H} F\right) .
$$

Because $\left.\bar{\omega}_{t} \in \Gamma_{Z^{\prime}}\right|_{\pi_{t}}$, it assigns the same value to all elements that belong to a given coset generated by the ideal $(Z-t \mathbf{1}) Z^{\prime}$. By definition in equation (21), for any value of $t, \vec{D}_{H}^{\prime}(t) F$ and $\vec{D}_{H} F$ are in the same coset relative to the ideal $(Z-t \mathbf{1}) Z^{\prime}$. Hence $\bar{\omega}_{t}\left(\vec{D}_{H} F\right)=\bar{\omega}_{t}\left(\vec{D}_{H}^{\prime}(t) F\right)$, from which (22) follows.

As demanded by property 2 of Proposition 2, the gauge flow of $C_{H}$ deparameterized by $(Z, \mathcal{F})$ projects to a dynamical flow on the unconstrained system defined by the fashionable algebra $\mathcal{F}$. The unconstrained dynamics on $\mathcal{F}$ can then be analyzed by the methods of conventional quantum mechanics. 


\subsection{Positivity}

For many applications it is also important to be able to reverse this process, linking positive states on a fashionable algebra at a given value of the clock with kinematical solutions to the constraint in $\Gamma$ and ultimately with physical states in $\Gamma_{C_{H}} / \sim_{C_{H}}$. Combining Definition 13 and Lemma 6, deparameterization is accomplished by finding a clock such that $\left[Z, C_{H}\right]=i \hbar \mathbf{1}$, and by splitting the kinematical algebra into subalgebras that share only the null element,

$$
\mathcal{A}=\mathcal{A} C_{H}+(Z-t \mathbf{1}) Z^{\prime}+\mathcal{F}
$$

where $\mathcal{F}$ is a $*$-subalgebra of $Z^{\prime}$ isomorphic to $Z^{\prime} /(Z-t \mathbf{1}) Z^{\prime}$ at each $t$.

Specifically, given a positive state $\tilde{\omega}$ on $\mathcal{F}$ and a value of the clock $t \in \mathbb{R}$, the corresponding relational state is $\bar{\omega}=\left.\psi_{t}^{-1}(\tilde{\omega}) \in \Gamma_{Z^{\prime}}\right|_{\pi_{t}}$, which by Lemma 7 is positive on $Z^{\prime}$. The corresponding solution of the constraint on $\mathcal{A}$ is $\omega=\phi^{-1}\left(\psi_{t}^{-1}(\tilde{\omega})\right) \in \Gamma_{C_{H}}$, which for any $B \in Z^{\prime}$ yields

$$
\omega((Z-t \mathbf{1}) B)=\bar{\omega}((Z-t \mathbf{1}) B)=0
$$

because $\left.\bar{\omega} \in \Gamma_{Z^{\prime}}\right|_{\pi_{t}}$. This, in turn, implies that

$$
\omega(Z)=t \quad \text { and } \quad \omega(Z B)=t \omega(B)=\omega(Z) \omega(B) .
$$

We find that this relation extends beyond $Z^{\prime}$ to the whole of $\mathcal{A}$ : Given any $A \in \mathcal{A}$, and any state $\omega \in \Gamma_{C_{H}}$, that satisfies $\omega(Z B)=\omega(Z) \omega(B)$ for all $B \in Z^{\prime}$, there are $B \in Z^{\prime}$ and $G \in \mathcal{A}$ such that

$$
\begin{aligned}
\omega(Z A) & =\omega\left(Z B+Z G C_{H}\right) \\
& =\omega(Z B) \\
& =\omega(Z) \omega(B) \\
& =\omega(Z) \omega\left(B+G C_{H}\right)=\omega(Z) \omega(A) .
\end{aligned}
$$

The following definition is therefore meaningful:

Definition 15 A state $\omega \in \Gamma$ is almost-positive with respect to a deparameterization of $C_{H}$ by $Z$ if

1. it annihilates the left ideal generated by $C_{H}: \omega\left(A C_{H}\right)=0$ for all $A \in \mathcal{A}$;

2. it is positive on the commutant of $Z: \omega\left(B B^{*}\right) \geq 0$ for all $B \in Z^{\prime}$;

3. it parameterizes left multiplication by $Z$ : for all $A \in \mathcal{A}, \omega(Z A)=\omega(Z) \omega(A)$.

The first condition ensures that $\omega \in \Gamma_{C_{H}}$ solves the constraint. The second condition ensures that $\omega$ restricts to some positive state on $Z^{\prime}$, and hence also on $\mathcal{F}$. The third condition ensures that this restriction belongs to the constant clock surface $\left.\Gamma_{Z^{\prime}}\right|_{\pi_{\omega(Z)}}$. In other words, 
Corollary 2 Every positive state on $\mathcal{F}$ has a unique extension to an almost-positive state $\omega$, for any given value of the clock $\omega(Z)=t \in \mathbb{R}$. Conversely, every almost positive state $\omega$ restricts to a positive relational state on $Z^{\prime}$ and hence a positive state on $\mathcal{F}$ at time $\omega(Z)$.

Remark: One way to interpret the last condition in Definition 15 is to notice that it requires fluctuations of $Z$ to vanish. For example, we have $\left(\Delta_{\omega} Z\right)^{2}:=\omega\left(Z^{2}\right)-\omega(Z)^{2}=0$. Just like a time parameter in ordinary quantum mechanics, $Z$ is sharply defined in such a state, but it does not correspond to an evolving observable since $Z$ is not an element of $\mathcal{F}$. As already noted in Section 2.4, the combination of almost-positivity and conditions required for deparameterization prevent $\omega$ from being positive on the whole of $\mathcal{A}$. The new notion of an almost-positive state, introduced here, may therefore be considered a maximal implementation of positivity in an internal-time setting, in which evolution is defined with respect to an algebra element. According to Lemma 7 , positivity of states can be extended from observables (realized here by $\mathcal{F}$ ) to time $\left(Z \in Z^{\prime}\right)$, but not to the full algebra $\mathcal{A}$.

According to Lemma 10, the gauge flow $\bar{S}_{C_{H}}(\lambda)$ generated by $C_{H}$ and mapped to $\Gamma_{Z^{\prime}}$ drags states from the subspace $\left.\Gamma_{Z^{\prime}}\right|_{\pi_{t}}$ to the subspace $\left.\Gamma_{Z^{\prime}}\right|_{\pi_{t+\lambda}}$ of gauge-fixed states.

Lemma 12 The gauge flow $S_{C_{H}}$ on $\Gamma$ drags an almost-positive state $\omega$ to another almostpositive state $S_{C_{H}}(\lambda) \omega$, such that $S_{C_{H}}(\lambda) \omega(Z)=\omega(Z)+\lambda$.

Proof: Lemma 4 guarantees that the flow remains on $\Gamma_{C_{H}}$. Since the adjoint action of $C_{H}$ is a $*$-compatible derivation on $Z^{\prime}$, according to Lemma 2 the corresponding flow maps states that are positive on $Z^{\prime}$ to other states that are positive on $Z^{\prime}$. For any almost-positive $\omega$, along the flow $\omega_{\lambda}:=S_{C_{H}}(\lambda) \omega$ we have

$$
\frac{\mathrm{d}}{\mathrm{d} \lambda} \omega_{\lambda}(Z)=\omega_{\lambda}\left(\vec{D}_{H} Z\right)=1
$$

Therefore, $\omega_{\lambda}(Z)=\omega(Z)+\lambda$.

To prove that parameterization of $Z$ is preserved along the flow, we follow the method of Lemma 2 and define a function $f_{A}(\lambda)=\omega_{\lambda}(Z A)-\omega_{\lambda}(Z) \omega_{\lambda}(A)$ for each $A \in \mathcal{A}$. Condition 3 of Definition 15 holds for $\omega_{\lambda}$ if and only if $f_{A}(\lambda)=0$ for all $A \in \mathcal{A}$. Suppose all of the functions $f_{A}\left(\lambda^{\prime}\right)=0$ for some $\lambda^{\prime}$. Taking an arbitrary $A \in \mathcal{A}$

$$
\begin{aligned}
\left.\frac{\mathrm{d}}{\mathrm{d} \lambda} \omega_{\lambda}(Z A)\right|_{\lambda=\lambda^{\prime}} & =\left.\left(\omega_{\lambda}\left(Z \vec{D}_{H} A\right)+\omega_{\lambda}(A)\right)\right|_{\lambda=\lambda^{\prime}} \\
& =\left.\omega_{\lambda^{\prime}}(Z) \frac{\mathrm{d}}{\mathrm{d} \lambda} \omega_{\lambda}(A)\right|_{\lambda=\lambda^{\prime}}+\left.\frac{\mathrm{d}}{\mathrm{d} \lambda} \omega_{\lambda}(Z)\right|_{\lambda=\lambda^{\prime}} \omega_{\lambda^{\prime}}(A) \\
& =\left.\frac{\mathrm{d}}{\mathrm{d} \lambda}\left(\omega_{\lambda}(Z) \omega_{\lambda}(A)\right)\right|_{\lambda=\lambda^{\prime}} .
\end{aligned}
$$

Consequently, $\mathrm{d} f_{A}(\lambda) / \mathrm{d} \lambda=0$ at $\lambda=\lambda^{\prime}$ for all $f_{A}(\lambda)$. Since $f_{A}(0)=\omega(Z A)-\omega(Z) \omega(A)=0$ for all $A \in \mathcal{A}$, it follows that $\left\{f_{A}(\lambda)=0, \forall \lambda\right\}_{A \in \mathcal{A}}$ is the solution to the flow generated by 
$C_{H}$. Hence $\omega_{\lambda}(Z A)=\omega_{\lambda}(Z) \omega_{\lambda}(A)$ remains true everywhere along the flow.

Since $\mathcal{A}_{\text {obs }}$ is not available in general, there is no full quantum analog of Proposition 11, But any available Dirac observable $O \in \mathcal{A}_{\text {obs }}$ is a valid observable with respect to any almost-positive state:

Lemma 13 If $O \in \mathcal{A}_{\mathrm{obs}}, \omega\left(O O^{*}\right) \geq 0$ for any almost-positive functional $\omega$ with respect to a deparameterization of $C_{H}$ by some $(Z, \mathcal{F})$.

Proof: Since $O \in \mathcal{A}_{\text {obs }} \subset \mathcal{A}$ is also an element of $\mathcal{A}$, the decomposition induced according to Definition 13 by deparameterization implies that we can write it as $O=A C_{H}+B$ for some $A \in \mathcal{A}$ and $B \in Z^{\prime}$. The fact that $O$ is in the commutant of $C_{H}$ implies $\left[O, C_{H}\right]=\left[B, C_{H}\right]+\left[A, C_{H}\right] C_{H}=0$. The second term on the left-hand side is in $\mathcal{A} C_{H}$, while equation (14) implies that the first term is in $Z^{\prime}$. Since the two subalgebras are linearly independent, the two terms must vanish separately, implying that $\left[B, C_{H}\right]=0$. Because $C_{H}$ is not a divisor of zero in a single constrained system, for $A \in \mathcal{A}$ we have that $A C_{H}=0$ implies $A=0$, and therefore $\left[A, C_{H}\right]=0$. These results also imply that $A^{*}$ and $B^{*}$ commute with $C_{H}$. Now suppose that $\omega$ is almost-positive with respect to deparameterization of $C_{H}$ by $Z$, then

$$
\begin{aligned}
\omega\left(O O^{*}\right) & =\omega\left(A C_{H} C_{H} A^{*}+B C_{H} A^{*}+A C_{H} B^{*}+B B^{*}\right) \\
& =\omega\left(\left(A A^{*} C_{H}+B A^{*}+A B^{*}\right) C_{H}\right)+\omega\left(B B^{*}\right) \\
& =\omega\left(B B^{*}\right) \geq 0
\end{aligned}
$$

because $B \in Z^{\prime}$, using $\omega(\mathcal{A C})=0$.

This completes the proof of Proposition 2 .

\subsection{Example: Parameterized Particle}

Let $\mathcal{A}$ be the polynomial algebra, generated by complex polynomials in the basic elements $Z, E, A_{i}$, with $i=1,2, \ldots, M$, and $\mathbf{1}\left(=: A_{0}\right)$. The generating elements are $*$-invariant, $Z=Z^{*}, E=E^{*}$ and $A_{i}=A_{i}^{*}$, and are subject to commutation relations $[Z, E]=i \hbar 1$, $\left[Z, A_{i}\right]=0=\left[E, A_{i}\right]$ and $\left[A_{i}, A_{j}\right]=i \hbar \sum_{k=0}^{M} \alpha_{i j}^{k} A_{k}$ for some $\alpha_{i j}^{k} \in \mathbb{C}$. This algebra is an example of an enveloping algebra of a Lie algebra, which we equip with the $\rho$-topology of [24.

For any $M$-tuple of integers $\vec{n}=\left(n_{1}, n_{2}, \ldots n_{M}\right)$, we define $A_{\vec{n}}=A_{1}^{n_{1}} A_{2}^{n_{2}} \ldots A_{M}^{n_{M}}$, with $A_{\overrightarrow{0}}:=1$. The set of monomials $\left\{A_{\vec{n}} Z^{m} E^{l}\right\}$ is linearly independent and forms a linear basis on $\mathcal{A}$. Let this system be subject to a single constraint of the form

$$
C_{H}=E+\mathrm{h}\left(Z, A_{i}\right),
$$

where $\mathrm{h}$ is a polynomial with an ordering such that $\mathrm{h}=\mathrm{h}^{*}$ and therefore $C_{H}=C_{H}^{*}$.

Consider the clock $(Z, \mathcal{F})$, where $\mathcal{F}$ is the algebra generated by all complex polynomials in just the elements $A_{i}$, and is therefore spanned by the linear basis of monomials $\left\{A_{\vec{n}}\right\}$. 
$Z=Z^{*}$ is given, and, since $Z$ commutes with itself and generators $A_{i}$, any element of $Z^{\prime}$ can be written as a linear combination of monomials of the form $A_{\vec{n}} Z^{m}$. The relational observables of this clock are given by the projection $\pi_{t}(A) \in Z^{\prime} /(Z-t \mathbf{1}) Z^{\prime}$ for any $A \in Z^{\prime}$, interpreted as " $A$ when $Z=t$." Here $\operatorname{ker} \pi_{t}=(Z-t \mathbf{1}) Z^{\prime}$, and it is easy to see that no nonzero elements of $(Z-t \mathbf{1}) Z^{\prime}$ are polynomials in generators $A_{i}$ alone, therefore $\mathcal{F} \cap$ ker $\pi_{t}=\{0\}$ as required by Definition 9 . For a basis monomial of $Z^{\prime}$ and any $t \in \mathbb{R}$

$$
\begin{aligned}
A_{\vec{n}} Z^{m} & =A_{\vec{n}}((Z-t \mathbf{1})+t \mathbf{1})^{m} \\
& =A_{\vec{n}} \sum_{k=0}^{m}\left(\begin{array}{c}
m \\
k
\end{array}\right)(Z-t \mathbf{1})^{k} t^{m-k} \\
& =A_{\vec{n}} t^{m}+A_{\vec{n}} \sum_{k=1}^{m}\left(\begin{array}{c}
m \\
k
\end{array}\right)(Z-t \mathbf{1})^{k} t^{m-k} .
\end{aligned}
$$

The last sum lies in the ideal $(Z-t \mathbf{1}) Z^{\prime}$, and, therefore, in the kernel of $\pi_{t}$; hence,

$$
\pi_{t}\left(A_{\vec{n}} Z^{m}\right)=\pi_{t}\left(A_{\vec{n}} t^{m}\right)=t^{m} \pi_{t}\left(A_{\vec{n}}\right) .
$$

Clearly, $\left\{\pi_{t}\left(A_{\vec{n}}\right)\right\}$ linearly spans $Z^{\prime} /(Z-t \mathbf{1}) Z^{\prime}$ for any $t \in \mathbb{R}$, so that $\pi_{t}(\mathcal{F})=Z^{\prime} /(Z-t \mathbf{1}) Z^{\prime}$. The pair $(Z, \mathcal{F})$ satisfy all requirements for a quantum clock given in Definition 9 ,

We now confirm that $(Z, \mathcal{F})$ deparameterizes $C_{H}$ according to Definition 13. We immediately see that $\left[Z, C_{H}\right]=[Z, E]=i \hbar \mathbf{1}$ as required. Since the expression for any non-zero element of $\mathcal{A} C_{H}$ in terms of the basis monomials of $\mathcal{A}$ includes at least one term of the form $A_{\vec{n}} Z^{m} E^{l}$ with $l \neq 0$, we infer $Z^{\prime} \cap \mathcal{A} C_{H}=\{0\}$. By substituting $E=C_{H}-\mathrm{h}\left(Z, A_{i}\right)$, we can write any element of $\mathcal{A}$ as a polynomial in $Z, C_{H}$, and $A_{i}$. Using the commutation relations, a factor of $C_{H}$ can be iteratively moved to the right whenever present, so that any $A \in \mathcal{A}$ can be written as

$$
A=\mathrm{p}_{0}\left(Z, A_{i}\right)+\mathrm{p}\left(Z, C_{H}, A_{i}\right) C_{H},
$$

for some polynomials $\mathrm{p}_{0}$ and $\mathrm{p}$. The first term is in $Z^{\prime}$, while the second is in $\mathcal{A} C_{H}$, the two sets therefore linearly generate the whole of $\mathcal{A}$. It is also immediately clear here that $Z^{\prime} \cup\left\{C_{H}\right\}$ algebraically generates $\mathcal{A}$ : iteratively moving every factor of $C_{H}$ to the right we can write

$$
A=\mathrm{p}_{1}\left(Z, A_{i}\right)+\mathrm{p}_{2}\left(Z, A_{i}\right) C_{H}+\ldots+\mathrm{p}_{N}\left(Z, A_{i}\right) C_{H}^{N} .
$$

According to Proposition 2 the flow generated by $C_{H}$ gives rise to a dynamical flow on the fashionables. To see this explicitly, we note that any $B \in Z^{\prime}$ can be written as a polynomial $\mathrm{p}\left(Z, A_{i}\right)$, so that the adjoint action of $C_{H}$ on $Z^{\prime}$ is given by

$$
\left[B, C_{H}\right]=[B, E]+[B, \mathrm{~h}]=\left.i \hbar \frac{\partial \mathrm{p}\left(s, A_{i}\right)}{\partial s}\right|_{s=Z}+\left[\mathrm{p}\left(Z, A_{i}\right), \mathrm{h}\left(Z, A_{i}\right)\right],
$$

both terms in the final expression are in $Z^{\prime}$. Since $\left[A_{\vec{n}}, E\right]=0$, restricting this to $F \in \mathcal{F}$ we have

$$
\vec{D}_{H} F=\frac{1}{i \hbar}\left[F, C_{H}\right]=\frac{1}{i \hbar}\left[F, \mathrm{~h}\left(Z, A_{\vec{n}}\right)\right] .
$$


The projection from $Z^{\prime}$ to $\mathcal{F}$ here has the explicit form

$$
\nu_{t} \circ \pi_{t}\left(A_{\vec{n}} Z^{m}\right)=\nu_{t}\left(t^{m} \pi_{t}\left(A_{\vec{n}}\right)\right)=A_{\vec{n}} t^{m},
$$

so that the commutator of two basis monomials projects as

$$
\nu_{t} \circ \pi_{t}\left(\left[A_{\vec{n}_{1}}, A_{\vec{n}_{2}} Z^{m}\right]\right)=\left[A_{\vec{n}_{1}}, A_{\vec{n}_{2}}\right] t^{m} .
$$

It follows that

$$
\vec{D}_{H}^{\prime}(t) F=\frac{1}{i \hbar}\left[F, \mathrm{~h}\left(t, A_{\vec{n}}\right)\right] .
$$

Therefore, this deparameterized example reduces the original constrained quantum system to an unconstrained quantum system with degrees of freedom generated by the basis $\left\{A_{\vec{n}}\right\}$, driven by the time-dependent Hamiltonian $\mathrm{h}\left(t, A_{\vec{n}}\right)$.

In this example, $\mathcal{A}_{\mathrm{obs}}=C_{H}^{\prime}$ contains $C_{H}$ itself and the identity element 1 . Any element of $\mathcal{A}_{\text {obs }}$ which is not a linear combination of a power of $C_{H}$ and $\mathbf{1}$ is a constant of motion of the (possibly time-dependent) Hamiltonian h. For most choices of a classical polynomial $h_{\text {class }}$, constants of motion which are $E$-independent and fulfill $\left\{O, h_{\text {class }}\right\}=0$, are generically non-polynomial, if they even exist in closed form [30, 31]. (The system may be non-integrable.) No quantization of such an observable can exist in our $\mathcal{A}$, and the available $\mathcal{A}_{\text {obs }}$ is incomplete. Even if one extends $\mathcal{A}$, for instance by using deformation quantization, in most cases of physical interest it is impossible to find a complete set of Dirac observables. Nevertheless, we have shown that it is possible to fix the gauge relative to $Z$ in any such system and uniquely specify physical states by relational observables, with the only requirement on $\mathrm{h}$ that $\mathrm{h} \in Z^{\prime}$ and $\mathrm{h}^{*}=\mathrm{h}$.

\subsection{Physical vs. relational states}

In Section 2.3 we defined physical states as orbits on $\Gamma_{C_{H}}$ of the entire collection of gauge flows generated by $\mathcal{A} C_{H}$, such that they are positive on the Dirac observables $\mathcal{A}_{\text {obs }}$. In Section 3.3 we established that relational states associated with a deparameterization of a constraint $C_{H}$ by a clock $(Z, \mathcal{F})$ are represented on $\Gamma_{C_{H}}$ by almost positive states of Definition 15. According to Lemma 13 each almost positive state is in the orbit corresponding to some physical state of the constraint. Furthermore, since time-evolution in $Z$ is generated by $C_{H}$, it is tangential to the gauge orbit so that all almost positive states along a time-evolution correspond to the same physical state. Deparameterization of Definition 13 interprets orbits of physical states (or, more accurately, a single preferred flow along these orbits) as time evolution relative to a physical clock. This relation leaves room for two important concerns.

First, some physical states may not be sampled by the deparameterization relative to a given clock $Z$ : It may happen that a physical orbit $[\omega]_{C_{H}}$ does not contain any state that parameterizes $Z$ as in the third condition of Definition [15. Stated differently, there is no guarantee that $\left.\phi\left([\omega]_{C_{H}}\right) \cap \Gamma_{Z^{\prime}}\right|_{\pi_{t}} \neq \emptyset$ for some $t \in \mathbb{R}$. While this concern warrants further investigation, it does not immediately appear to be physically problematic: since a clock is 
a physical part of the constrained system, the ability to assign simultaneous definite values to the clock and its commutant should in general require special states. At present, we do not claim that every physical state can be captured by deparameterization relative to a given clock.

Second, a given deparameterization may suffer from the analog of Gribov problems in gauge theories if a physical state is sampled by multiple relational states, in other words, if $\left.\phi\left([\omega]_{C_{H}}\right) \cap \Gamma_{Z^{\prime}}\right|_{\pi_{t}}$ contains more than one state for some $t \in \mathbb{R}$. If this happens, each of those states will give rise to a time-evolution relative to $Z$, and a single physical state will be associated with multiple inequivalent evolving states on $\mathcal{F}$. However only the states that are positive on $Z^{\prime}$ have a physically useful relational interpretation (see discussion in Section 2.4 and 3.3 ). Therefore this possibility constitutes an ambiguity of physical relational states only if $\left.\phi\left([\omega]_{C_{H}}\right) \cap \Gamma_{Z^{\prime}}\right|_{\pi_{t}}$ contains more than one state that is positive on $Z^{\prime}$. An indication that such physical ambiguity is avoided by deparameterization comes from the following consideration. One would expect that multiple intersections of two $\left.\Gamma_{Z^{\prime}}\right|_{\pi_{t}}$ by the orbit $\phi\left([\omega]_{C_{H}}\right)$ would be generated by following a $Z^{\prime}$-positivity-preserving gauge flow. From equation (16) (together with (13)), we see that the only gauge flow that, when mapped onto $\Gamma_{Z^{\prime}}$, is generated by a $*$-compatible derivation, and therefore preserves positivity on $Z^{\prime}$, is the one generated by $C_{H}$ itself. For this particular flow we note the following result.

Lemma 14 Let $C_{H}$ be deparameterized by a clock $(Z, \mathcal{F})$. Then, each one-parameter family of states in $\Gamma_{Z^{\prime}}$ along the constraint flow generated by $C_{H}$ intersects each constant time surface at most once.

Proof: Along the flow of $C_{H}$ we have $\mathrm{d} \bar{\omega}_{\lambda}(Z) / \mathrm{d} \lambda=\bar{\omega}_{\lambda}\left(\vec{D}_{H} Z\right)=1 \neq 0$. Therefore, $\bar{\omega}_{\lambda}(Z)$ is monotonic in $\lambda$, and any two states $\bar{\omega}_{\lambda_{1}}$ and $\bar{\omega}_{\lambda_{2}}$ with $\lambda_{1} \neq \lambda_{2}$ along this flow assign different values to $Z-t \mathbf{1} \in \operatorname{ker} \pi_{t}$ for any given $t \in \mathbb{R}$. It is, therefore, not possible for both states to belong to the same constant time surface $\left.\Gamma_{Z^{\prime}}\right|_{\pi_{t}}$.

\section{General polynomial constraints}

A general constraint element $C$ is not immediately of the form required for deparameterization to exist. While the kinematical algebraic structure of most model theories that are of interest to quantum gravity and quantum cosmology possesses clocks $Z$ that are not constant along the constraint flow, that is, $[Z, C] \neq 0$, the condition $[Z, C]=i \hbar \mathbf{1}$ (or $[Z, C]=a \mathbf{1}$ with $a \in \mathbb{C})$ is not usually satisfied. For instance, most Hamiltonian constraints in such systems are quadratic in momenta, resulting in $[Z,[Z, C]] \neq 0$ for a clock

$Z$, as in the scalar example of cosmological models with energy density (2). There are also examples of constraints for which $[Z, C] \in Z^{\prime}$ but not a multiple of the unit. 


\subsection{Linearization and cancellation}

In some of these cases, it may be possible to "linearize" the constraint by finding a suitable $C_{H} \in \mathcal{A}$ which satisfies all three criteria of a deparameterization with respect to $Z$ and has a gauge flow and a constraint surface related to those of $C$ : If $N \in \mathcal{A}$ is such that $C=N C_{H}$ and $C_{H}$ as in Definition 13, we have $\mathcal{A}_{C} \subset \mathcal{A}_{C_{H}}$ and therefore $\Gamma_{C_{H}} \subset \Gamma_{C}$. Moreover, $\omega \sim_{C} \psi$ if $\omega \sim_{C_{H}} \psi$. If $N$ is invertible in $\mathcal{A}, \Gamma_{C_{H}}=\Gamma_{C}$ and $\sim_{C_{H}}=\sim_{C}$, but this case is too restrictive for most practical purposes.

Example: The constraint $C=E^{2}-\mathrm{h}\left(A_{i}\right)^{2}$ with $Z$-independent $\mathrm{h}=\mathrm{h}^{*}$ can be factorized as $C=(E-\mathrm{h})(E+\mathrm{h})=N C_{H}$ with $N=E-\mathrm{h}$ and $C_{H}=E+\mathrm{h}$. We have $\left[N, C_{H}\right]=0$, but $N$ does not have an inverse. However, if $\omega \in \Gamma_{C_{H}}$ and $\omega(E) \neq 0$ it follows that $\omega(N) \neq 0$, in which case it may be of interest to study evolution of the state with respect to $C_{H}$. More generally, we define $C_{ \pm}=E \pm \mathrm{h}\left(A_{i}\right)$ such that

$$
C=C_{+} C_{-}=C_{-} C_{+}
$$

Since either $\omega\left(A C_{+}\right)=0$ or $\omega\left(A C_{-}\right)=0$ also imply $\omega(A C)=0$, every left solution of $C_{ \pm}$ is also a left solution of $C$. Therefore both constraint surfaces $\Gamma_{C \pm}$ are contained within the constraint surface $\Gamma_{C}$. Furthermore, normalized combinations of states from $\Gamma_{C+}$ and $\Gamma_{C-}$ also give us solutions to $C$. In particular, for any $a_{i}^{(+)}, a_{i}^{(-)} \in \mathbb{C}, \omega_{i}^{(+)} \in \Gamma_{C+}$, and $\omega_{i}^{(-)} \in \Gamma_{C-}$

$$
\omega=\sum_{i} a_{i}^{(+)} \omega_{i}^{(+)}+\sum_{i} a_{i}^{(-)} \omega_{i}^{(-)}
$$

is a left solution of $C$, which is normalized provided that $\left(\sum_{i} a_{i}^{(+)}+\sum_{i} a_{i}^{(-)}\right)=1$.

In this example the two subsets $\Gamma_{C \pm}$ are not disjoint. A solution to both constraint factors must satisfy $\omega\left(A C_{+}\right)=0$ and $\omega\left(A C_{-}\right)=0$ for any $A \in \mathcal{A}$. These conditions are equivalent to requiring that both $\omega(A E)=0$ and $\omega(A \mathrm{~h})=0$ for all $A \in \mathcal{A}$, since

$$
\omega(A E)=\omega\left(A \frac{1}{2}\left(C_{+}+C_{-}\right)\right)=\frac{1}{2}\left(\omega\left(A C_{+}\right)+\omega\left(A C_{-}\right)\right)=0,
$$

and similarly

$$
\omega(A \mathrm{~h})=\omega\left(A \frac{1}{2}\left(C_{+}-C_{-}\right)\right)=\frac{1}{2}\left(\omega\left(A C_{+}\right)-\omega\left(A C_{-}\right)\right)=0 .
$$

Conversely, $\omega(A E)=0$ and $\omega(A \mathrm{~h})=0$ imply $\omega\left(A C_{+}\right)=0$ and $\omega\left(A C_{-}\right)=0$. The only restriction on the values assigned by a kinematical state $\omega \in \Gamma$, in addition to continuity, is normalization $\omega(\mathbf{1})=1$. It is therefore possible to satisfy both $\omega(A E)=0$ and $\omega(A \mathrm{~h})=0$ for all $A$, unless $A E+B \mathrm{~h}=\mathbf{1}$ for some $A, B \in \mathcal{A}$. No such $A$ and $B$ exist within our polynomial $\mathcal{A}$, hence the intersection $\Gamma_{C+} \cap \Gamma_{C-}$ is non-empty. However if we consider only the states that are positive on $Z^{\prime}$, as required by Definition [15, there may be additional 
restrictions: suppose $\mathrm{h}=F F^{*}+\epsilon_{0} \mathbf{1}$ for some $F \in \mathcal{F}$ and real $\epsilon_{0}>0$. Then, for any normalized state that is positive on $\mathcal{F}$

$$
\omega(\mathrm{h})=\omega\left(F F^{*}\right)+\epsilon_{0} \geq \epsilon_{0}>0
$$

which means $\omega(\mathrm{h})=0$ cannot be satisfied. Hence depending on $\mathrm{h}$ the sets of almostpositive states with respect to internal clock $Z$ defined by the two constraint factors may be disjoint.

Using the original constraint $C$, the orbits are generated by the subalgebra $\mathcal{A} C$, as opposed to $\mathcal{A} C_{ \pm}$if we use one of the factors instead. Neither of the two factors has an inverse already contained within $\mathcal{A}$ (the only element with an explicit inverse in $\mathcal{A}$ here is 1). Thus $\mathcal{A} C$ is a proper subset of $\mathcal{A} C_{ \pm}$, and hence the original orbits of $C$ are contained within the larger orbits of $C_{ \pm}$. This guarantees, via Lemma 4, that a physical state of the original constraint $C$ is either entirely inside $\Gamma_{C \pm}$ or entirely outside of it. However, some gauge flows generated by the factor constraints $C_{ \pm}$are not gauge orbits of $C$ and can potentially link distinct gauge orbits of the original constraint $C$. Therefore, a physical state with respect to $C_{ \pm}$generally corresponds to a region of the space of physical states with respect to the original constraint $C$.

This complication would not arise if $N$ had an inverse in $\mathcal{A}$. However, even if $N$ is non-invertible there are in general some states on which its action can be "reversed" in the following sense.

Definition 16 Left multiplication of $A \in \mathcal{A}$ can be canceled in $\omega \in \Gamma$ if for any $B \in \mathcal{A}$, $\omega(G A B)=0$ for all $G \in \mathcal{A}$ implies $\omega(G B)=0$ for all $G \in \mathcal{A}$.

This state-by-state condition differs from the algebraic cancellation property. In our concrete example, only the zero element is a divisor of zero in $\mathcal{A}$. However, even though $C B=0$ implies $B=0$, left multiplication by $C$ cannot be canceled in any of its left solutions $\omega \in \Gamma_{C}$. Setting $B=\mathbf{1}$, we get $\omega(G C \mathbf{1})=\omega(G C)=0$ for all $G \in \mathcal{A}$, which is not equivalent to $\omega(G \mathbf{1})=0$ for all $G \in \mathcal{A}$, since setting $G=\mathbf{1}$ would violate normalization.

Definition $17 A$ constraint $C$ is deparameterized by factorization with respect to an internal clock $Z$, if there are $N, C_{H} \in \mathcal{A}$, such that (i) $C=N C_{H}$, (ii) there is at least one state $\omega \in \Gamma_{C_{H}}$ in which left multiplication by $N$ can be canceled, and (iii) $C_{H}=C_{H}^{*}$ is deparameterized by $(Z, \mathcal{F})$.

In our concrete example, if we deparameterize our system using $C_{+}$as the constraint, we consider only the states $\omega \in \Gamma_{C_{+}}$in which the left multiplication of $C_{-}$can be canceled. (In particular, this means that $\omega \notin \Gamma_{C_{-}}$.)

Lemma 15 For a constraint that is deparameterized by factorization as $C=N C_{H}$, for any $A \in \mathcal{A}_{\mathrm{obs}}$ of $C$ and $\omega \in \Gamma_{C_{H}}$ such that left multiplication by $N$ can be canceled in $\omega$, the value $\omega(A)$ is invariant along all of the gauge flows generated by $C_{H}$. 
Proof: We first observe that, since $[A, C]=0$, in particular $\omega(B[A, C])=0$ for any $B \in \mathcal{A}$. Which means

$$
\begin{aligned}
0 & =\omega\left(B\left[A, N C_{H}\right]\right) \\
& =\omega\left(B N\left[A, C_{H}\right]\right)+\omega\left(B[A, N] C_{H}\right)=\omega\left(B N\left[A, C_{H}\right]\right)
\end{aligned}
$$

Since this holds for any $B$, cancellation of left multiplication by $N$ in $\omega$ implies that

$$
\omega\left(B\left[A, C_{H}\right]\right)=0, \quad \text { for all } B \in \mathcal{A} .
$$

The above property holds along all of the gauge flows generated by $C_{H}$. To see this let us fix an arbitrary $G \in \mathcal{A}$ and, following the method of Lemma 2, define functions $f_{B}(\lambda)=S_{G C_{H}} \omega\left(B\left[A, C_{H}\right]\right)$ for each $B \in \mathcal{A}$. Clearly, $f_{B}(0)=0$ for all $B \in \mathcal{A}$. Suppose all functions $f_{B}\left(\lambda^{\prime}\right)=0$ for some $\lambda^{\prime}$, then

$$
\begin{aligned}
\left.i \hbar \frac{\mathrm{d} f_{B}}{\mathrm{~d} \lambda}\right|_{\lambda=\lambda^{\prime}} & =\left.i \hbar \frac{\mathrm{d}}{\mathrm{d} \lambda}\left(S_{G C_{H}}(\lambda) \omega\left(B\left[A, C_{H}\right]\right)\right)\right|_{\lambda=\lambda^{\prime}} \\
& =S_{G C_{H}}\left(\lambda^{\prime}\right) \omega\left(B\left[A, C_{H}\right] G C_{H}-G C_{H} B\left[A, C_{H}\right]\right) \\
& =S_{G C_{H}}\left(\lambda^{\prime}\right) \omega\left(B\left[A, C_{H}\right] G C_{H}\right)-f_{G C_{H} B}\left(\lambda^{\prime}\right)=0
\end{aligned}
$$

where we used the fact that $S_{G C_{H}}\left(\lambda^{\prime}\right) \omega \in \Gamma_{C_{H}}$ according to Lemma 4. We see that $\left\{f_{B}(\lambda)=0, \forall \lambda\right\}$ is the solution of the dynamical flow generated by $G C_{H}$ that agrees with our initial conditions. Since $G$ was arbitrary, $S_{G C_{H}}(\lambda) \omega\left(B\left[A, C_{H}\right]\right)=0$ for all $B, G \in \mathcal{A}$ and $\lambda \in \mathbb{R}$.

Using the above result, the value of $\omega(A)$ along the gauge flow generated by $B C_{H}$, with arbitrary $B \in \mathcal{A}$, varies according to

$$
\begin{aligned}
i \hbar \frac{\mathrm{d}}{\mathrm{d} \lambda}\left(S_{B C_{H}}(\lambda) \omega(A)\right) & =S_{B C_{H}}(\lambda) \omega\left(\left[A, B C_{H}\right]\right) \\
& =S_{B C_{H}}(\lambda) \omega\left(B\left[A, C_{H}\right]\right)+S_{B C_{H}}(\lambda) \omega\left([A, B] C_{H}\right)=0, \text { for all } \lambda .
\end{aligned}
$$

Therefore, using gauge flows generated by $C_{H}$ does not affect the values assigned to the set of Dirac observables of the original constraint $C$, so long as we use states on which left multiplication of the factor $N$ can be canceled. In this section's example, the roles of $C_{+}$ and $C_{-}$can be reversed since the two factors commute.

In principle this construction also applies to a constraint that can be written as a product of non-commuting factors, as one would expect in the case of time-dependent Hamiltonians $\mathrm{h}\left(A_{i}, Z\right)$. However, factorizing such a constraint is much more complicated. Example: If we factorize a constraint of the form $C=E^{2}-H^{2}$ with $[E, H] \neq 0$, we have $C \neq(E-H)(E+H)$, but we can try to find $X \in Z^{\prime}$ such that $C=(E-H+X)(E+H-X)$. Multiplying the two factors, we have

$$
C=E^{2}-H^{2}-X^{2}+[E, H]-[E, X]+[X, H]+2 H X
$$


provided that

$$
2 H X=[H, E]+[H, X]+[E, X]+X^{2} .
$$

This equation has a formal power-series solution $X=\sum_{n=1}^{\infty}(i \hbar)^{n} X_{n}$ with

$$
2 H X_{1}=\frac{[H, E]}{i \hbar}
$$

and

$$
2 H X_{n}=\frac{\left[H, X_{n-1}\right]}{i \hbar}+\frac{\left[E, X_{n-1}\right]}{i \hbar}+\sum_{a=1}^{n-1} X_{n-a} X_{a} .
$$

We can split $X=\frac{1}{2}\left(X_{+}+X_{-}\right)$into its $*$-invariant and anti-*-invariant contributions, $X_{+}=\frac{1}{2}\left(X+X^{*}\right)$ and $X_{-}=\frac{1}{2}\left(X-X^{*}\right)$, and define

$$
H^{\prime}=H-X_{+} \quad \text { and } \quad E^{\prime}=E-X_{-} .
$$

As in the example with commuting factors, $H^{*}=H^{\prime}$ and $\left[Z, E^{\prime}\right]=[Z, E]=i \hbar \mathbf{1}$ but $E^{\prime *} \neq E^{\prime}$. There are therefore almost-positive states, but the gauge flow of $C_{H}=E^{\prime}+H^{\prime}$ does not induce a $*$-compatible derivation, unless it so happens that $X_{-}=0$.

For a systematic analysis of suitable factorizations, we need to carefully consider the adjointness conditions imposed on the factors of the constraint.

\subsection{Adjointness relations}

The adjointness relation $C^{*}=C$ imposed on constraints guarantees that $\mathcal{A}_{\text {obs }} \subset \mathcal{A}$ inherits a $*$-relation, which in turn makes it possible to define physical states as positive linear functionals on $\mathcal{A}_{\text {obs }}$. This condition also restricts possible factorization choices that could be applied to linearize constraints. Suppose a constraint $C=C^{*}$ can be written as $C=N C_{H}$, where $N$ can be algebraically canceled within $\mathcal{A}$ and $C_{H}=C_{H}^{*}$ allows a deparameterization with respect to $Z^{*}=Z \in \mathcal{A}$. Then $C$ can be deparameterized with respect to $Z$ by factorization, using the same method as we applied to $C=C_{-} C_{+}$to cast a subset of its physical states as dynamical evolution in $Z$. Under these conditions, $C_{H}$ uniformizes the flow generated by $C$ : Since $[Z, C]=i \hbar N$, we may consider $N$ as the "non-constant rate" of evolution determined by $C$, while evolution with respect to $C_{H}$ has constant rate.

In order to satisfy $C=C^{*}$ we need $N C_{H}=C_{H} N^{*}$, which can be rewritten as

$$
\left[N, C_{H}\right]=C_{H}\left(N^{*}-N\right) .
$$

If the non-constant rate is required to be real when evaluated in a positive state $\omega$, we need $N^{*}=N$. In this case, (23) implies $\left[N, C_{H}\right]=0$, such that the rate is, in fact, constant on solutions of the constraint because $N$ is a constant of motion with respect to $C_{H}$. Conversely, if $\left[N, C_{H}\right]=0$ we obtain $\left(N-N^{*}\right) C_{H}=0$, and if $C_{H}$ can be algebraically canceled within $\mathcal{A}$, we get $N=N^{*}$. These cases constitute two sufficient conditions for factorization to result in a deparameterization. 
Provided that the clock is part of a canonical pair, $[Z, E]=i \hbar \mathbf{1}$, as in the example from the previous subsection, the most general form of a factorizable constraint is $C=N(E+H)$,

where $H=H^{*}$ commutes with $Z$, and condition (23) holds for $C_{H}=E+H$. Further properties depend on the $E$-dependence of $C$.

\subsubsection{Non-relativistic constraints}

Definition $18 A$ constraint $C \in \mathcal{A}$ is non-relativistic of rate $N \in \mathcal{A}$ if there is a canonical generator $E \in \mathcal{A}$ conjugate to $Z \in \mathcal{A},[Z, E]=i \hbar \mathbf{1}$, such that $[Z, C]=i \hbar N \in Z^{\prime}$.

Definition 19 A non-relativistic constraint $C \in \mathcal{A}$ is of constant flow rate $N \in \mathcal{A}$ if there is a $C_{H} \in \mathcal{A}$ such that $C=N C_{H}$ and $\left[N, C_{H}\right]=0$.

Lemma 16 Every deparameterizable non-relativistic constraint is of constant flow rate.

Proof: $\quad$ Since $C^{*}=C$ and $Z^{*}=Z$ imply $[Z, C]^{*}=-[Z, C]$, we immediately obtain $N^{*}=N$ from $N=[Z, C] /(i \hbar)$. Using this in (23), we have $\left[N, C_{H}\right]=0$.

Remark: The condition $\left[N, C_{H}\right]=0$ of constant flow rate shows the restrictive nature of adjointness conditions: Only constants of motion with respect to $C_{H}$ are allowed as factors of $E$ in non-relativistic constraints. Written as $[N, E]=-[N, H]$ if $C_{H}=E+H$, the condition amounts to a partial differential equation for $N$ as a function of $Z$ and the remaining canonical variables.

Lemma 17 If a non-relativistic constraint $C$ is deparameterizable, it is of the form $C=$ $A_{1} E+A_{0}$ such that $A_{1}=A_{1}^{*}$ and $\left[A_{0}, A_{1}\right]=A_{1}\left[A_{1}, E\right]$.

Proof: $\quad$ Since the constraint is non-relativistic, it is linear in $E$ and can be written as $C=A_{1} E+A_{0}$ with $A_{1}$ and $A_{0}$ such that $\left[Z, A_{1}\right]=\left[Z, A_{0}\right]=0$. The conditions $C=C^{*}$ and $Z=Z^{*}$ imply that $[Z, C]^{*}=-[Z, C]$, and thus $A_{1}=A_{1}^{*}$. Since $A_{1}$ plays the role of the factor $N$, it must be a left factor of $A_{0}$ : There must exist $H \in \mathcal{A}$ such that $A_{0}=A_{1} H$ and

$$
C=A_{1}(E+H) .
$$

In order for $C$ to be deparameterizable, according to Lemma 16, the flow rate $A_{1}=N$ must be constant with respect to $C_{H}=E+H$. Therefore, $\left[A_{1}, E\right]=-\left[A_{1}, H\right]$, which, upon left multiplication with $A_{1}$, implies $A_{1}\left[A_{1}, E\right]=-\left[A_{1}, A_{0}\right]$ because $A_{0}=A_{1} H$.

Remark: The condition $H=H^{*}$, obtained from $C_{H}^{*}=C_{H}$ for a deparameterizable constraint, implies

$$
A_{0}^{*}=H A_{1}=A_{0}+\left[A_{1}, H\right] .
$$

Therefore, $A_{0}$ in $C=A_{1} E+A_{0}$ is not self-adoint unless $A_{1}$ commutes with $H$. 
Remark: If, in spite of Lemma 16, we try to factorize a constraint of non-constant flow rate, we end up with a non-self-adjoint $C_{H}$. To see this, consider a non-relativistic constraint of the form $C=\frac{1}{2}\left(B_{1} E+E B_{1}\right)+B_{0}$ with $B_{0}=B_{0}^{*}$ and invertible $B_{1}=B_{1}^{*}$, we can write

$C=B_{1} E+B_{0}-\frac{1}{2}\left[B_{1}, E\right]=B_{1}\left(E+\frac{1}{2}\left(B_{1}^{-1} B_{0}+B_{0} B_{1}^{-1}\right)+\frac{1}{2}\left[B_{1}^{-1}, B_{0}\right]-\frac{1}{2} B_{1}^{-1}\left[B_{1}, E\right]\right)$.

Defining $N=B_{1}$,

$$
H=\frac{1}{2}\left(B_{1}^{-1} B_{0}+B_{0} B_{1}^{-1}\right)
$$

and

$$
E^{\prime}=E+\frac{1}{2}\left[B_{1}^{-1}, B_{0}\right]-\frac{1}{2} B_{1}^{-1}\left[B_{1}, E\right]
$$

we can write $C=N C_{H}$ with $C_{H}=E^{\prime}+H$. It follows that $H=H^{*}$, and $\left[Z, E^{\prime}\right]=[Z, E]=$ $i \hbar$ since $\left[Z, B_{1}\right]=0$ and $\left[Z, B_{0}\right]=0$ for a non-relativistic constraint. However,

$$
E^{\prime *}=E-\frac{1}{2}\left[B_{1}^{-1}, B_{0}\right]+\frac{1}{2}\left[B_{1}, E\right] B_{1}^{-1} \neq E^{\prime}
$$

and therefore $C_{H}^{*} \neq C_{H}$. For $\omega \in \Gamma_{C_{H}}$, we have $\omega\left(E^{\prime}\right)=-\omega(H) \in \mathbb{R}$. If $\omega$ is almostpositive, this equation is consistent even though $E^{\prime} \neq E^{\prime *}$ while $H^{*}=H$ : because $E^{\prime} \notin Z^{\prime}$, an almost-positive state may take on a real value in a non-self-adjoint $E^{\prime}$. However, the gauge flow of $C_{H} \neq C_{H}^{*}$ does not induce a $*$-preserving derivation on any fashionable algebra $\mathcal{F} \subset Z^{\prime}$ because, in general, $\left[f, E^{\prime}\right] \neq 0$ for $f \in \mathcal{F}$ unless $B_{0}$ and $B_{1}$ are multiples of the unit.

\subsubsection{Relativistic constraints}

Definition 20 A constraint $C$ is relativistic if there is a canonical generator $E$ conjugate to $Z,[Z, E]=i \hbar \mathbf{1}$, such that $0 \neq[Z,[Z, C]] \in Z^{\prime}$.

A relativistic constraint that is deparameterizable by factorization has the form

$$
C=\left(N_{1} E+N_{0}\right)(E+H)=N_{1} E^{2}+\left(N_{0}+N_{1} H\right) E+N_{1}[E, H]+N_{0} H
$$

where $H^{*}=H$, and $\left[Z, N_{1}\right]=\left[Z, N_{0}\right]=[Z, H]=0$. Using $C^{*}=C$ and $Z=Z^{*}$, we have

$$
\left(\frac{1}{i \hbar}\left[Z, \frac{1}{i \hbar}[Z, C]\right]\right)=\left(\frac{1}{i \hbar}\left[Z, \frac{1}{i \hbar}[Z, C]\right]\right)^{*},
$$

which quickly yields $N_{1}=N_{1}^{*}$. The flow rate of $C$ with respect to $C_{H}=E+H$ is given by $N_{1} E+N_{0}$, such that $C=N C_{H}$. In contrast to linear or relativistic constraints, the flow rate depends on $E$. 
Lemma 18 If a relativistic constraint $C$ that is deparameterizable by factorization is of constant real flow rate $N$, it is of the form $C=N C_{H}$ with $N=N_{1} E+N_{0}$ and $C_{H}=E+H$ such that

$$
\begin{gathered}
N_{0}^{*}=N_{0}+\left[N_{1}, E\right], \\
{\left[N_{1}, E\right]+\left[N_{1}, H\right]=0,}
\end{gathered}
$$

and

$$
N_{1}[H, E]=\left[N_{0}, E\right]+\left[N_{0}, H\right]
$$

Proof: For real flow rate, $N=N^{*}$ implies $N^{*}=E N_{1}+N_{0}^{*}=N$ and therefore (24). Constant flow rate, $\left[N, C_{H}\right]=0$, results in

$$
0=\left(\left[N_{1}, E\right]+\left[N_{1}, H\right]\right) E+N_{1}[E, H]+\left[N_{0}, E\right]+\left[N_{0}, H\right] .
$$

Taking a commutator with $Z$ on both sides, only the term proportional to $E$ survives giving us (25). Substituting this back into (27) results in (26).

The three conditions of Lemma 18 together are sufficient to make the quadratic constraint deparameterizable by factorization.

Lemma $19 A$ relativistic constraint with real constant flow rate $N=E+N_{0}$ is of the form $C=\tilde{E}^{2}-h$ such that $\tilde{E}^{*}=\tilde{E}$ and $h^{*}=h$ as well as $[Z, \tilde{E}]=i \hbar \mathbf{1}$ and $[Z, h]=0$.

Proof: A relativistic constraint with flow rate $N=E+N_{0}$, using $N_{1}=\mathbf{1}$ in terms Lemma [18, can be written as $C=E^{2}+A_{1} E+A_{0}$, where $\left[A_{i}, Z\right]=0$. Using the notation of Lemma 18,

$$
A_{1}=N_{0}+H \quad \text { and } \quad A_{0}=[E, H]+N_{0} H .
$$

We have $A_{1}^{*}=A_{1}$ because $N_{0}^{*}=N_{0}$ from equation (24). Equation (25) is trivially satisfied, while (26) becomes

$$
[H, E]=\left[N_{0}, E\right]+\left[N_{0}, H\right]
$$

We rewrite

$$
\begin{aligned}
C & =\left(E^{2}+\frac{1}{2}\left(A_{1} E+E A_{1}\right)+\frac{1}{4} A_{1}^{2}\right)-\frac{1}{4} A_{1}^{2}+\frac{1}{2}\left[A_{1}, E\right]+A_{0} \\
& =\left(E+\frac{1}{2} A_{1}\right)^{2}-\left(\frac{1}{4} A_{1}^{2}-\frac{1}{2}\left[A_{1}, E\right]-A_{0}\right) \\
& =\tilde{E}^{2}-h
\end{aligned}
$$

setting $h=\frac{1}{4} A_{1}^{2}-\frac{1}{2}\left[A_{1}, E\right]-A_{0}$ and $\tilde{E}=E+\frac{1}{2} A_{1}$. Using (28) and (29), we compute

$$
\begin{aligned}
h & =\frac{1}{4} A_{1}^{2}-\frac{1}{2}\left[N_{0}, E\right]+\frac{1}{2}[H, E]-N_{0} H \\
& =\frac{1}{4} A_{1}^{2}-\frac{1}{2}\left[N_{0}, E\right]+\frac{1}{2}\left(\left[N_{0}, E\right]+\left[N_{0}, H\right]\right)-N_{0} H \\
& =\frac{1}{4} A_{1}^{2}-\frac{1}{2}\left(N_{0} H+H N_{0}\right)
\end{aligned}
$$


such that $[Z, h]=0$. By inspection, $h^{*}=h$ as well as $\tilde{E}^{*}=\tilde{E}$. Moreover, since $\left[Z, A_{1}\right]=0$, we have $[Z, \tilde{E}]=[Z, E]=i \hbar \mathbf{1}$.

Lemma 20 A relativistic constraint of the form $C=(E+g)^{2}-h$, such that $g^{*}=g$, $h^{*}=h$, and $[Z, g]=[Z, h]=0$, is deparameterizable by factorization only if $[E+g, h]=0$.

Proof: The factorized version of such a constraint must be of the form

$$
C=\left(E+N_{0}\right)(E+H)=E^{2}+\left(N_{0}+H\right) E+[E, H]+N_{0} H
$$

which we compare with

$$
(E+g)^{2}-h=E^{2}+g^{2}+2 g E+[E, g]-h .
$$

Taking a commutator with $Z$ and equating the two expressions yields $g=\frac{1}{2}\left(N_{0}+H\right)$. Using this result to eliminate $g$ and setting the two expressions equal gives

$$
[E, H]+N_{0} H=\frac{1}{4}\left(N_{0}+H\right)^{2}+\frac{1}{2}\left[E, N_{0}\right]+\frac{1}{2}[E, H]-h .
$$

This expression can be rearranged to solve for $h$ in terms of $H, N_{0}$, and their commutators with $E$

$$
h=\frac{1}{2}\left([H, E]+\left[E, N_{0}\right]\right)+\frac{1}{2}\left(N_{0}^{2}+H^{2}+2 N_{0} H-\left[N_{0}, H\right]\right)-N_{0} H .
$$

We combine the first two terms using equation (26) $\left(\right.$ with $\left.N_{1}=\mathbf{1}\right)$ :

$$
\begin{aligned}
h & =\frac{1}{2}\left[N_{0}, H\right]+\frac{1}{4} N_{0}^{2}+\frac{1}{4} H^{2}-\frac{1}{2} N_{0} H-\frac{1}{4}\left[N_{0}, H\right] \\
& =\frac{1}{4}\left(N_{0}^{2}+H^{2}-2 N_{0} H+\left[N_{0}, H\right]\right)=\frac{1}{4}\left(N_{0}-H\right)^{2} .
\end{aligned}
$$

Now consider the commutator

$$
\begin{aligned}
{\left[E+g, N_{0}-H\right] } & =\left[E+\frac{1}{2}\left(N_{0}+H\right), N_{0}-H\right] \\
& =[H, E]+\left[E, N_{0}\right]-\frac{1}{2}\left[N_{0}, H\right]+\frac{1}{2}\left[H, N_{0}\right] \\
& =\left[N_{0}, H\right]+\left[H, N_{0}\right]=0,
\end{aligned}
$$

where in the final equality we once again used (26). This result immediately implies

$$
[E+g, h]=\left[E+g, \frac{1}{4}\left(N_{0}-H\right)^{2}\right]=0
$$

as a necessary condition for our constraint to be deparameterizable by factorization. 
Example: We assume that $h=\sqrt{h}^{2}$ has a square root $\sqrt{h}=\sqrt{h}^{*}$ in $\mathcal{A}$. Comparison of the two constraint forms results in

$$
g=\frac{1}{2}\left(N_{0}+H\right), \quad \text { and } \quad \sqrt{h}=\frac{1}{2}\left(N_{0}-H\right) .
$$

The factorizability condition (26) now gives

$$
2[E, \sqrt{h}]=[\sqrt{h}, g]-[g, \sqrt{h}],
$$

or

$$
i \hbar \frac{\partial \sqrt{h}}{\partial Z}=[\sqrt{h}, g]
$$

For example, in a two-component system with canonical generators $[Z, E]=[q, p]=i \hbar \mathbf{1}$, setting

$$
\sqrt{h}=p+\frac{1}{2}\left(q^{2}-Z^{2}\right), \quad \text { and } g=Z(q-Z),
$$

satisfies equation (30) and leads to the factorization

$$
\begin{aligned}
C & =(E+Z(q-Z))^{2}-\left(p+\frac{1}{2}\left(q^{2}-Z^{2}\right)\right)^{2} \\
& =\left(E+p+\frac{1}{2} q^{2}-\frac{3}{2} Z^{2}+q Z\right)\left(E-\left(p+\frac{1}{2}(q-Z)^{2}\right)\right) .
\end{aligned}
$$

As this example demonstrates, in general a constraint $C \in \mathcal{A}$ has to be of a specific form in order for a deparameterization and therefore evolution with respect to a gauge section to exist. This result showcases the power of our general approach to quantum dynamical reduction. The restrictions of the type found in Lemmas 18 20 have not been anticipated by the standard method of deparameterization on a fixed Hilbert space, which treats each specific scenario individually and has mainly been applied to time-independent systems in which $C=N C_{H}$, where $N$ and $C_{H}$ commute. The additional restrictions derived here are the consequence of the inclusion of time dependence from the outset, as well as the general algebraic treatment that is not tied to a specific Hilbert-space representation.

\section{Acknowledgements}

This work was supported in part by NSF grants PHY-1607414 and PHY-1912168. 


\section{References}

[1] J. E. Marsden and A. Weinstein, Reduction of symplectic manifolds with symmetry, Rep. Math. Phys. 5 (1974) 121-130

[2] M. Bojowald and T. Strobl, Poisson Geometry in Constrained Systems, Rev. Math. Phys. 15 (2003) 663-703, hep-th/0112074

[3] K. V. Kuchař, Time and interpretations of quantum gravity, In G. Kunstatter, D. E. Vincent, and J. G. Williams, editors, Proceedings of the 4th Canadian Conference on General Relativity and Relativistic Astrophysics, Singapore, 1992. World Scientific

[4] C. J. Isham, Canonical Quantum Gravity and the Question of Time, In J. Ehlers and H. Friedrich, editors, Canonical Gravity: From Classical to Quantum, pages 150-169. Springer-Verlag, Berlin, Heidelberg, 1994

[5] P. A. M. Dirac, Generalized Hamiltonian dynamics, Can. J. Math. 2 (1950) 129-148

[6] W. F. Blyth and C. J. Isham, Quantization of a Friedmann universe filled with a scalar field, Phys. Rev. D 11 (1975) 768-778

[7] C. Rovelli, Time in Quantum Gravity: An Hypothesis, Phys. Rev. D 43 (1991) $442-456$

[8] B. Dittrich, Partial and Complete Observables for Hamiltonian Constrained Systems, Gen. Rel. Grav. 39 (2007) 1891-1927, gr-qc/0411013

[9] B. Dittrich, Partial and Complete Observables for Canonical General Relativity, Class. Quant. Grav. 23 (2006) 6155-6184, gr-qc/0507106

[10] F. Bayen, M. Flato, C. Fronsdal, A. Lichnerowicz, and D. Sternheimer, Deformation theory and quantization. I. Deformation of Symplectic Structures, Ann. Phys. 111 (1978) 61-110

[11] F. Bayen, M. Flato, C. Fronsdal, A. Lichnerowicz, and D. Sternheimer, Deformation theory and quantization. II. Physical Applications, Ann. Phys. 111 (1978) 111-151

[12] M. Kontsevich, Deformation quantization of Poisson manifolds, I, arXiv:q-alg/9709040

[13] T. Damour, M. Henneaux, and H. Nicolai, Cosmological Billiards, Class. Quantum Grav. 20 (2003) R145-R200, hep-th/0212256

[14] M. Bojowald, Loop Quantum Cosmology, Living Rev. Relativity 11 (2008) 4, gr-qc/0601085, http://www.livingreviews.org/lrr-2008-4

[15] M. Bojowald, Quantum cosmology: a review, Rep. Prog. Phys. 78 (2015) 023901, arXiv:1501.04899. 
[16] M. Bojowald, Large scale effective theory for cosmological bounces, Phys. Rev. D 75 (2007) 081301(R), gr-qc/0608100

[17] J. Ben Achour and E. Livine, The Thiemann Complexifier and the CVH algebra for Classical and Quantum FLRW Cosmology, Phys. Rev. D 96 (2017) 066025, arXiv:1705.03772

[18] J. Ben Achour and E. Livine, Polymer Quantum Cosmology: Lifting quantization ambiguities using a $\mathrm{SL}(2, \mathbb{R})$ conformal symmetry, Phys. Rev. D 99 (2019), arXiv:1806.09290]

[19] J. Ben Achour and E. Livine, Protected SL $(2, \mathbb{R})$ Symmetry in Quantum Cosmology, JCAP 09 (2019) 012, arXiv:1904.06149

[20] M. Bojowald, Non-bouncing solutions in loop quantum cosmology, JCAP 07 (2020) 029, arXiv:1906.02231]

[21] B. S. DeWitt, Quantum Theory of Gravity. I. The Canonical Theory, Phys. Rev. 160 (1967) 1113-1148

[22] M. Günaydin and B. Zumino, Magnetic charge and non-associative algebras, In Symposium to honor G. C. Wick, Pisa, Italy, 1984

[23] R. Jackiw, Three-Cocycle in Mathematics and Physics, Phys. Rev. Lett. 54 (1985) 159

[24] J.-P. Jurzak, Simple facts about algebras of unbounded operators, J. Func. Anal. 21 (1976) 469-482

[25] R. Haag, Local Quantum Physics, Springer-Verlag, Berlin, Heidelberg, New York, 1992

[26] T. L. Gill and W. W. Zachary, Foundations for Relativistic Quantum Theory I: Feynman's Operator Calculus and the Dyson Conjectures, J. Math. Phys. 43 (2002) 69, math-ph/0405059

[27] M. Bojowald, P. A. Höhn, and A. Tsobanjan, An effective approach to the problem of time, Class. Quantum Grav. 28 (2011) 035006, arXiv:1009.5953.

[28] M. Bojowald, P. A. Höhn, and A. Tsobanjan, An effective approach to the problem of time: general features and examples, Phys. Rev. D 83 (2011) 125023, arXiv:1011.3040,

[29] P. A. Höhn, E. Kubalova, and A. Tsobanjan, Effective relational dynamics of a nonintegrable cosmological model, Phys. Rev. D 86 (2012) 065014, arXiv:1111.5193

[30] B. Dittrich, P. A. Hoehn, T. A. Koslowski, and M. I. Nelson, Chaos, Dirac observables and constraint quantization, arXiv:1508.01947] 
[31] B. Dittrich, P. A. Hoehn, T. A. Koslowski, and M. I. Nelson, Can chaos be observed in quantum gravity?, Phys. Lett. B 769 (2017) 554-560, arXiv:1602.03237.

[32] F. Giacomini, A. Castro-Ruiz, and C. Brukner, Quantum mechanics and the covariance of physical laws in quantum reference frames, Nat. Commun. 10 (2019) 494, arXiv:1712.07207,

[33] A. Vanrietvelde, P. A. Hoehn, F. Giacomini, and E. Castro-Ruiz, A change of perspective: switching quantum reference frames via a perspective-neutral framework, Quantum 4 (2020) 225, arXiv:1809.00556]

[34] A. Vanrietvelde, P. A. Hoehn, and F. Giacomini, Switching quantum reference frames in the $N$-body problem and the absence of global relational perspectives, arXiv:1809.05093

[35] P. A. Hoehn, A. R. H. Smith, and M. P. E. Lock, The Trinity of Relational Quantum Dynamics, arXiv:1912.00033 